\title{
Prácticas directivas y competencias de los altos directivos para una gestión efectiva en el Estado de Chile
}

\author{
Carlos Castro
}

Universidad de Chile

\section{Resumen}

Este estudio de caso, con características principalmente exploratorias, contempla el uso de métodos y técnicas cuantitativas y cualitativas de análisis, con el propósito de hacer aportes nuevos y significativos en el estudio de la gestión de los Altos Directivos Públicos del Estado en Chile, considerando su realidad cultural e institucional. El principal instrumento para el levantamiento de información primaria, corresponde a una encuesta electrónica a la totalidad de los directivos públicos de primer y segundo nivel jerárquico seleccionados por el SADP, luego de un extenso proceso previo de validación. Posteriormente, a través de un focus group con directivos de primer nivel jerárquico, se busca validar y contrastar resultados, para concluir con implicancias de política pública en pos de mejorar el Sistema y potenciar nuevas líneas de investigación en el área de la gestión pública. La relevancia de esta investigación se sustenta en la trascendencia e impacto de la calidad de un directivo público a nivel organizacional y en el Estado. En esta misma línea, la literatura demuestra la importancia del liderazgo y la calidad del directivo, así como el rol central de la profesionalización de directivos públicos en cualquier proceso de reforma del Estado de un país.

Palabras clave: Altos Directivos Públicos, gestión gerencial, liderazgo.

Management practices and competencies of senior managers for effective management in the State of Chile

\begin{abstract}
This paper has a primarily exploratory feature, which contemplates the use of quantitative and qualitative methods and analysis techniques, with the purpose to develop new and significant contributions to the study of management for the Senior Public Servers of Chile (SADP), considering its cultural and institutional reality. The main instrument for the collection of primary data is an electronic survey to all public managers of first and second hierarchical level selected by the SADP, after an extensive validation process. Subsequently, through a focus group with managers of first hierarchical level, seeks to validate and contrast results, concluding with implications for public policy towards improving the system and enhancement of new lines of research in the area of public management. The relevance of this research is based on the importance and impact of the quality of public management at the organizational level in the State. In the same vein, the literature shows the importance of leadership and quality of managers as well as the central role of the professionalization of public management reform process in any country.
\end{abstract}

Keywords: Senior Public Management, managerial management, leadership.

*Dirección de correspondencia [Correspondence

address]: Carlos Castro, Universidad de Chile

E-mail: ccastro@dii.uchile.cl 


\section{Introducción}

El estudio de la gestión pública y la calidad de los altos directivos públicos es un tema relevante pues está íntimamente ligado al desarrollo del país. Como plantean Waissbluth et al. (2008), la calidad de los directivos debe constituir una prioridad fundamental de una adecuada política pública transversal respecto a la gestión del Estado.

Salvador Valdés (2001), aplicando las estimaciones de Evans y Rauch (1999), concluye que si Chile hubiera tenido los directivos públicos de la calidad de los de Hong Kong durante el período 1970-1990, el crecimiento económico nacional hubiese sido superior 1,5 puntos porcentuales del PIB cada año, y de efectuar la comparación con Singapur, el crecimiento hubiese sido superior en 2,1 puntos porcentuales del PIB anuales, es decir, aproximadamente entre 3.000 y 4.000 millones de dólares al año. Demostrando de esta manera la incidencia de la calidad de la institucionalidad pública en el crecimiento económico de cada país.

El Estado de Chile, a través de la creación del Servicio Civil, ha respondido a la problemática de la administración del Estado y ha adoptado los aprendizajes internacionales. La elección meramente política de los altos cargos públicos ha sido reemplazada por un sistema que regula gran parte de la selecciones de manera meritocrática, asegurando un estándar competitivo con menor discrecionalidad en los procesos de selección.

El Servicio Civil se preocupa principalmente de: (i) la selección de altos directivos públicos, (ii) los procesos de capacitación y apoyo, y (iii) los convenios de desempeño. No obstante el notable esfuerzo de profesionalización, este sistema ha establecido definiciones en el diseño de sus procesos sin contar con un conocimiento suficiente sobre cuáles son las competencias genéricas pertinentes para la función de los cargos y la relación de estos atributos con el desempeño de las instituciones públicas.

Diseñar perfiles, sistemas de formación o apoyo y mecanismos de control del desempeño sin saber con la profundidad necesaria quiénes son y cómo funcionan estos directivos, implica serios riesgos potenciales.

Con mayor evidencia empírica acerca del desempeño de los altos directivos se podría aportar a una mejora sustancial del Servicio Civil en su diseño, implementación y operación, derivando en una mejor gestión del estado y por lo tanto en mayor bienestar para el país. Por estos motivos, este estudio pretende recabar la evidencia empírica planteada, tratando de responder a las preguntas: ¿Quiénes son, cómo son y qué hacen los directivos públicos chilenos? ¿Qué es lo que hace que un directivo público sea efectivo en el Estado chileno?

Mejorar los macro-sistemas de gestión de personas del Estado, está intrínsecamente relacionado al nivel de desarrollo de los países y a la capacidad que tienen estos de ir modernizándose. Si bien existen modelos y perfiles que se han diseñado y construido en el último medio siglo, la alta dirección del país debe responder a las necesidades e idiosincrasia de cada sociedad por lo que la generación de conocimientos interna es muy valiosa.

\section{La gestión gerencial y el liderazgo}

La administración de las organizaciones ha sido un objeto de estudio de las ciencias sociales a partir del desarrollo de la industria post Revolución Industrial. A finales del siglo XIX se adopta el concepto gerencia, para definir a aquellos que administran y ejecutan el actuar de organizaciones productivas. Posteriormente se desarrollaron principios fundamentales de la gerencia y su relación con la economía, la política y la sociedad. Un autor fundamental en este desarrollo fue Peter Drunker, quien definió la gerencia como "el factor determinante de las organizaciones, cuya principal función es contribuir al trabajo en equipo y al fortalecimiento de la eficiencia" (2001: 480).

Posteriormente, Henry Mintzberg (2009a) desarrolló el concepto de práctica gerencial. El autor entiende que la gerencia no es por sí sola una ciencia ni una profesión, sino que es una práctica, y por lo tanto se aprende a través de la experiencia. Mintzberg propone un triángulo virtuoso compuesto de oficio (experiencia acumulada), ciencia (conocimiento acumulado) y arte (capacidad de producir a partir de la intuición). El resultado de la conjugación de estas tres variables, cada una en su justa medida según sea el caso, será un trabajo que es por encima de todo: una práctica.

El autor le resta valor al conocimiento en sí mismo, dándole mayor importancia al conocimiento de otros dentro de la organización. El acento no está solo en la gestión de sí mismo de un gerente, sino también cómo este puede aprovechar (gestionar) las personas que se desempeñan bajo él. El gerente se ubica al centro del modelo; entre la unidad organizacional, por la cual él es formalmente responsable, y el entorno, compuesto por el resto de la organización y todo lo que está fuera de ella. Como muestra la Ilustración 1, "la gerencia se ejerce en tres planos, desde lo conceptual hasta lo concreto: con información, por medio de las personas y directamente hasta la acción" (Mintzberg, 2009a: 62-63). 
Figura 1: "Un modelo de la Práctica Gerencial"

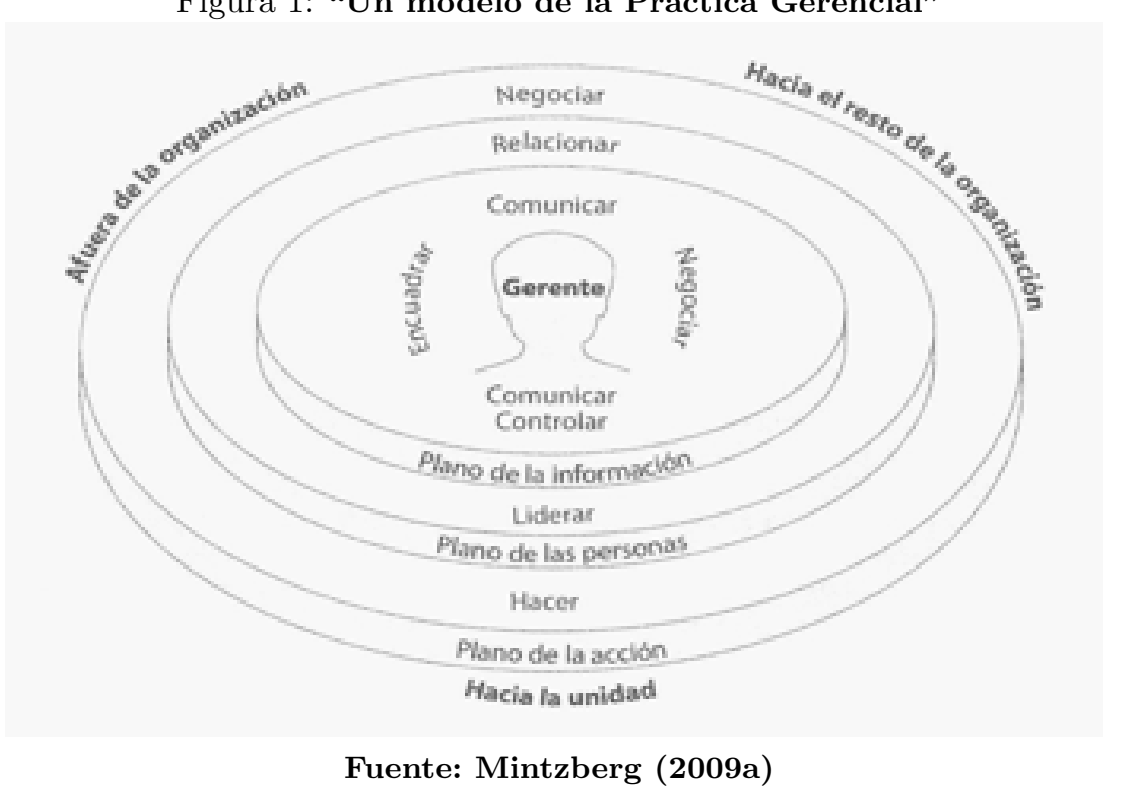

En aproximaciones de la literatura contemporánea se ha tendido a homogenizar los conceptos de gerencia y liderazgo. Mintzberg diferencia ambos conceptos, sin hacerlos excluyentes, sino complementarios. En esta investigación se entiende el liderazgo como la habilidad del directivo público para influenciar a los miembros de una organización con el fin de orientar sus comportamientos hacia la consecución de los fines de dicha institución.

El mayor estudio empírico desarrollado a la fecha sobre competencias y prácticas de liderazgo, es el esfuerzo transcultural del Global Leadership and Organizational Behaviour Effectiveness Research Program, GLOBE (2004) . Este estudio, ha listado comprensivamente 382 atributos y comportamientos de líderes organizacionales, resumidos en 112 ítems de un cuestionario aplicado a 17.300 personas, de diferentes industrias y niveles, en 951 organizaciones y 62 culturas y sociedad distintas . Las ?prácticas? son entendidas en el sentido de cómo se hacen las cosas y a su vez los ?valores? son los juicios respecto cómo deben ser hechas las cosas. De esta manera, se agruparon las características de los líderes en las siguientes 6 "dimensiones globales": i) basados en carisma y valores; ii) orientados a la construcción de equipos; iii) orientados a la participación de los trabajadores; iv) con enfoque hacia "lo humano"; v) orientados a la "autonomía"; vi) autoprotegido. Una de conclusiones más relevantes es que en Latinoamérica el liderazgo preferido (juicio de valor, no necesariamente la práctica) es aquel orientando a la construcción de equipo; en tanto en países anglosajones, es más deseable el liderazgo carismático y basado en valores (Centro de Sistemas Públicos, 2012).
En cuanto a las prácticas de gestión de los directivos, Mintzberg (1975) concluye que se pueden organizar en tres grandes ámbitos: a) relaciones interpersonales; b) gestión de la información; c) gestión de las decisiones. Las principales competencias necesarias para desarrollar dichas funciones son: persuadir personas, diseminar información, analizar información, generar procesos de toma de decisiones (muchas veces con poca información). Los directivos trabajan de modo discontinuo y no lineal, en un sentido contrario a las hipótesis que suponía un proceso guiado por el análisis, planificación, ejecución y control. Estos estarían orientados a la acción y poco a la reflexión. En el caso de los directivos estudiados (5), más de la mitad de sus actividades no duraron más de 9 minutos, y solo 1 actividad duró más de una hora. Además, los directivos en general se harían cargo de una serie de rutinas protocolares, de relaciones públicas, de negociación, de procesamiento de información soft que vincula a su organización con el entorno. Los directivos obtendrían información en el $80 \%$ de su tiempo mediante contactos orales y no por escrito u otros medios. Les importa más la información informal y menos la información agregada.

En el sector público, se suman nuevos desafíos al rol que debe cumplir un directivo, como gestionar su entorno político, potenciar las capacidades internas organizacionales y estar en reflexión permanente sobre el propósito de la institución, entre otras variables. Estos desafíos debiesen acompañarse de una gestión del cambio efectiva al interior de las organizaciones, no obstante, la teoría sobre este tópico desarrollada por John Kotter (1995) concluye que la mayoría de los esfuerzos por gestionar el cambio tienen resultados mediocres. Las causas 
que explican esta situación son: (1) los responsables del cambio no generarían suficiente convicción estratégica en la cúpula y en el conjunto de actores claves de la organización; (2) no se genera una visión seductora de los resultados del cambio que estimule, motive y compense las dificultades propias de un proceso de esta naturaleza; (3) no hay suficiente comunicación y discusión con la organización de la estrategia; (4) hay escasa programación de evidencias de logros de corto plazo o quick wins; y (5) falta perseverancia en los procesos de implementación o cambio, los cuales son normalmente largos y operativamente tediosos e implican una dedicación no menor de tiempo de los directivos personalmente para ir ajustando los procesos (Centro de Sistemas Públicos, 2012). En este contexto el desarrollo de una práctica gerencial como la planteada por Mintzberg se vuelve aún más compleja, pero necesaria y desafiante a la vez.

Kotter, Mintzberg y otros íconos del management, ambicionan que los modelos de gestión que han desarrollado sean efectivos en los diversos escenarios que le toca enfrentar al directivo. Por ello resulta útil comprender que la gestión efectiva de una organización en el contexto de Estado debe "combinar potencias y debilidades porque trabajan sobre una diversidad de temas sociales, políticos y económicos. [?] No es solo una cuestión de eficiencia y productividad. Es efectiva por cuanto no existen fisuras en el frente interno (cohesión) y se genere suficiente confianza en los actores externos (credibilidad)" (Etkin, 2005). En contraste, Shepherd (1999) destaca el valor del uso de las jerarquías en aquellos países que han seguido la dirección de la Nueva Gestión Pública, corriente basal para destacar que los sistemas modernos de gestión se fundan en una gestión efectiva, compuesta por un conjunto de elementos como liderazgo, recursos, incentivos, libertad de gestión y valores congruentes (World Bank, 1997)

Gran parte de las teorías expuestas se podrían alojar en el paradigma posburocrático de Barzelay (1998), donde uno de los pilares fundamentales se sustenta en fomentar mayor participación, autonomía y autoridad de los directivos públicos, para un mejor aprovechamiento de sus conocimientos, capacidades y esfuerzos. Por consiguiente, el robustecimiento del servicio civil como gran gestor de las personas en el Estado, es clave para una mirada de largo plazo que genere los incentivos, la estabilidad y la profesionalización.

\section{Marco institucional y normativo del Sistema de Alta Dirección Pública}

El Sistema de Alta Dirección Pública se origina a partir del documento "Acuerdos PolíticoLegislativos para la Modernización del Estado, la Transparencia y la Promoción del Crecimiento" publicado a fines de enero de 2003, sin embargo las primeras ideas sobre un Sistema de Alta Dirección Pública surgieron alrededor de 1998 (Centro de Políticas Públicas UC, 2013). El documento nombrado fue el producto de un diagnóstico compartido por amplios sectores políticos y sociales del país, ante ausencia de un mecanismo competitivo y selectivo basado en el mérito (Lambertini y Silva, 2009). A su vez el clima político generado por algunos casos de probidad y transparencia de la época , propició el impulso de la Agenda de Modernización del Estado en acuerdo con los partidos políticos de gobierno y oposición.

El diseño de este Sistema se inspiró especialmente en las experiencias de Nueva Zelanda y Reino Unido, las cuales fueron maduradas en reflexiones realizadas principalmente en centros chilenos de investigación y estudios (Valdés, 2001).

A mediados de 2003 se promulga la Ley 19.882, que regula la nueva política de personal a los funcionarios públicos y crea: la Dirección Nacional del Servicio Civil y el Consejo de Alta Dirección Pública. Como bien sintetiza el informe del Centro de Políticas Públicas UC (2013), "esta ley planteó una separación de las funciones de diseño y ejecución o implementación de las políticas públicas dentro de los Ministerios. Para el caso de los Servicios que implementan políticas públicas, se definieron perfiles de selección para el primer y segundo nivel jerárquico, y estos cargos se establecieron como sujetos a un proceso de selección meritocrático, acorde al perfil requerido".

La Dirección Nacional del Servicio Civil se compone de dos Subdirecciones, la Subdirección de Alta Dirección Pública, desarrolla e implementa acciones para el funcionamiento del Sistema de Alta Dirección Pública y la Subdirección de Desarrollo de las Personas, que diseña e implementa acciones y políticas para avanzar hacia una gestión estratégica y más eficiente de los recursos humanos de la administración civil del Estado .

\section{Subdirección de Alta Dirección Pública}

Esta unidad está encargada de velar por el buen funcionamiento y la transparencia del proceso de concursos públicos que ejecuta el Sistema de Alta 
Dirección Pública, que implica el aseguramiento del mérito y las competencias de los futuros directivos, así como también el resguardo de la información del proceso y custodiar el principio de no discriminación en él.

En la actualidad, la gestión de personas en el Estado en su rango más alto ha quedado en manos del Servicio Civil, que aplica el proceso de selección a través del SADP a 1255 cargos, pertenecientes a los servicios públicos adscritos y a otros organismos públicos, que si bien no están obligados por ley, seleccionan algunos de sus cargos directivos a través del Sistema. De los cargos anteriormente nombrados, corresponden 944 a aquellos adscritos normativamente, siendo 110 de Primer Nivel Jerárquico, fundamentalmente jefes de servicio y 834 de Segundo Nivel Jerárquico, entre ellos directores regionales, jefes de división o equivalentes . Es importante destacar que las diferencias entre los niveles jerárquicos de los Servicios, dependerá de cada institución y no se relacionan a la Escala Única de Sueldos.

Los periodos de nombramiento de los altos directivos son de tres años en el cargo, con dedicación exclusiva, pudiendo ser renovados hasta dos veces más y cada período con la misma duración de tres años. No obstante lo anterior, pueden ser removidos en cualquier momento sin expresión de causa, ya que la ley establece que estos cargos son de confianza de la autoridad correspondiente, independiente del proceso de concurso que debe realizarse para su selección.

\section{Subdirección de Desarrollo de las Personas}

La Dirección Nacional del Servicio Civil tiene por misión "fortalecer la capacidad de los servicios públicos en el desarrollo e implementación de políticas y prácticas de gestión de personas, para aumentar la productividad del sector público y la calidad de los bienes y servicios que requiere la ciudadanía [?]Con este propósito, la Subdirección de Desarrollo de las Personas es la encargada de supervisar, coordinar y perfeccionar la gestión de personas en la administración central del Estado, mediante el diseño de políticas de gestión de personas, la promoción de reformas para el mejoramiento de la gestión, la administración y provisión de información para la función pública, la promoción de buenas prácticas y climas laborales gratos y estimulantes, y el desarrollo de asesorías para autoridades de gobierno y jefes de servicio, siguiendo los principios del mérito, la equidad y la productividad, y en el marco de una implementación descentralizada".

\section{Perfil del Alto Directivo Público}

El artículo cuadragésimo noveno de la Ley 19.882, establece el marco para definir el perfil del cargo de los Altos Directivos Públicos: Los ministros respectivos deberán definir los perfiles profesionales y de competencias y aptitudes que deberán cumplir los candidatos a los cargos de jefes superiores de servicio. Estos perfiles deberán ser aprobados por el Consejo de Alta Dirección Pública y ser enviados a la Dirección Nacional del Servicio Civil para su registro. En el caso de los altos directivos públicos del segundo nivel jerárquico de la respectiva institución, corresponderá a los jefes superiores de servicio definir dichos perfiles.

El informe del Centro de Políticas Públicas UC (2013) plantea al respecto que

"los perfiles publicados contienen requisitos legales (educación universitaria, experiencia previa comprobable), misión del cargo, contexto del cargo, entre otros. La evaluación se realiza en función de un conjunto de atributos para el ejercicio del cargo, que incluye visión estratégica, gestión y logro, relación con el entorno y articulación de redes, manejo de crisis y contingencias, liderazgo, innovación y flexibilidad, y conocimientos técnicos. Además, el perfil contiene una descripción de funciones estratégicas, las que se refieren a funciones permanentes del cargo, y los desafíos del cargo, que son las funciones de contingencia que deberá el directivo hacer frente al inicio de su periodo. Al respecto, el Servicio Civil plantea que el perfil debiese ser un insumo fundamental para la elaboración del convenio de desempeño". 


\section{Metodología}

En este estudio de caso se utilizó una metodología mixta de carácter exploratorio y descriptivo. Se dividió en tres partes, cada una de ellas buscando responder a distintos objetivos mediante diversos métodos de investigación.

Buscando responder a la pregunta ¿qué insumos son necesarios para poder diseñar los instrumentos y qué cosas son necesarias de levantar?, se revisó literatura de temas referentes al management y gerencia, liderazgo, gestión pública, reforma del Estado en Chile y gestión efectiva; e información secundaria tales como literatura institucional y normativa, prensa, sitios web de servicios públicos y bases de datos del servicio civil. Se participó de seminarios de la temática a investigar y se llevaron a cabo entrevistas semiestructuradas, conversaciones exploratorias y un cuestionario preliminar de medición a Altos Directivos Públicos. Para la observación de los directivos se usó la técnica no estructurada desarrollada por Mintzberg (2009b).

A partir de conceptos provenientes de Mintzberg (2009b), se construyó una encuesta de manera participativa a través de discusión con grupos entendidos en la materia, reuniones académicas con investigadores del Centro de Sistemas Públicos y reuniones sobre la práctica del SADP con el Servicio Civil. La encuesta se aplicó de manera online a la totalidad de directivos públicos electos bajo SADP de 1er y 2do nivel. Posteriormente se construyó y depuró la base de datos con los datos provenientes del Servicio Civil.

Por último se llevaron a cabo focus group siguiendo los pasos de la Guía Conceptual y Metodológica de la Universidad de El Bosque (Escobar y Bonilla, 2009).

\section{Resultados}

\section{Caracterización de la Muestra}

Se obtuvo una alta tasa de respuesta considerando el medio electrónico utilizado. Además la representación del primer nivel jerárquico es porcentualmente mayor a la media de todas las respuestas.

Al hacer un análisis estadístico de la representatividad de la muestra, se presenta el obstáculo de que el universo con el que se cuenta es relativamente pequeño en términos estadísticos a la hora de hablar de una "población". En la literatura se encuentra que entre menor sea el tamaño de la po- blación (N), más alta debe ser la muestra (n). Por tanto, no es posible validar la representatividad de la muestra, así que se calculó el margen de error al extrapolar la muestra a la población. Esto se puede hacer ya que se conoce el tamaño de la población $\mathrm{N}$ y el tamaño de la muestra n. Se definirá un nivel de confianza (z) determinado al $95 \%$ con lo que se puede averiguar, como hicimos antes con la fórmula para poblaciones infinitas, el margen de error (e) con el que se puede extrapolar los resultados a todo el universo (Morales, 2012). La fórmula usada para calcular este margen es de:

$$
e=\sqrt{\frac{\left(p q z^{2}\right)(N-n)}{n(N-1)}}
$$

Se establece un margen de 5,53\% de error, al extrapolar la muestra al universo muestral, lo que es considerablemente razonable para un universo pequeño. Asimismo, se pudo apreciar, al depurar la data obtenida y diseñar la base de datos con que finalmente se trabajó la información, que existió una confusión respecto a la declaración del nivel jerárquico al que pertenecían los directivos encuestados, principalmente en el segundo nivel. Esto se corrigió mediante criterios de reemplazo, con aquellos que marcaron la opción "Otro" y declararon ser un tercer nivel jerárquico u otras denominaciones similares. Esto suele ocurrir, pues un error común es asociar el nivel jerárquico a la Escala Única de Sueldos (EUS) de cada institución.

A partir de la declaración de las Subsecretarías de algunos Servicios, se puede observar la desagregación de la tasa de respuesta anterior por Ministerio y por Nivel Jerárquico. También el porcentaje de cobertura en relación al número de directivos que compone el universo de cada institución ministerial, lo que se muestra en la siguiente tabla.

No fue una buena aproximación preguntar para la identificación de los encuestados, por la Subsecretaría a la que pertenecen. En general se dieron respuestas disonantes entre directivos que se desempeñan en el mismo Servicio, vinculándose a distintas Subsecretarías en el caso de que hubiese más de una o bien declaraciones de autonomía al interior del Ministerio. Esto ocurre principalmente porque las leyes que crean los Servicios, vinculan a la institución y su Jefe Superior con el Presidente de la República, mediante un Ministerio, no a través de una Subsecretaría establecida. A ratos esto pudiese confundirse pues la supervigilancia del Servicio por parte del Ministro, en la mayoría de los casos recae en el Subsecretario.

En una pregunta abierta sobre funciones principales que cumplía el servicio en el que se desem- 
Tabla 1: Tasa de respuesta encuesta según Nivel Jerárquico

\begin{tabular}{|llll|}
\hline Nivel & Muestra & Universo & Tasa respuesta \\
\hline I & 29 & 79 & $36,7 \%$ \\
\hline II & 178 & 526 & $33,8 \%$ \\
\hline Total & $\mathbf{2 0 7}$ & $\mathbf{6 0 5}$ & $\mathbf{3 4 , 2} \%$ \\
\hline
\end{tabular}

Fuente: Elaboración propia

Tabla 2: Cantidad de respuestas por Ministerio y Nivel

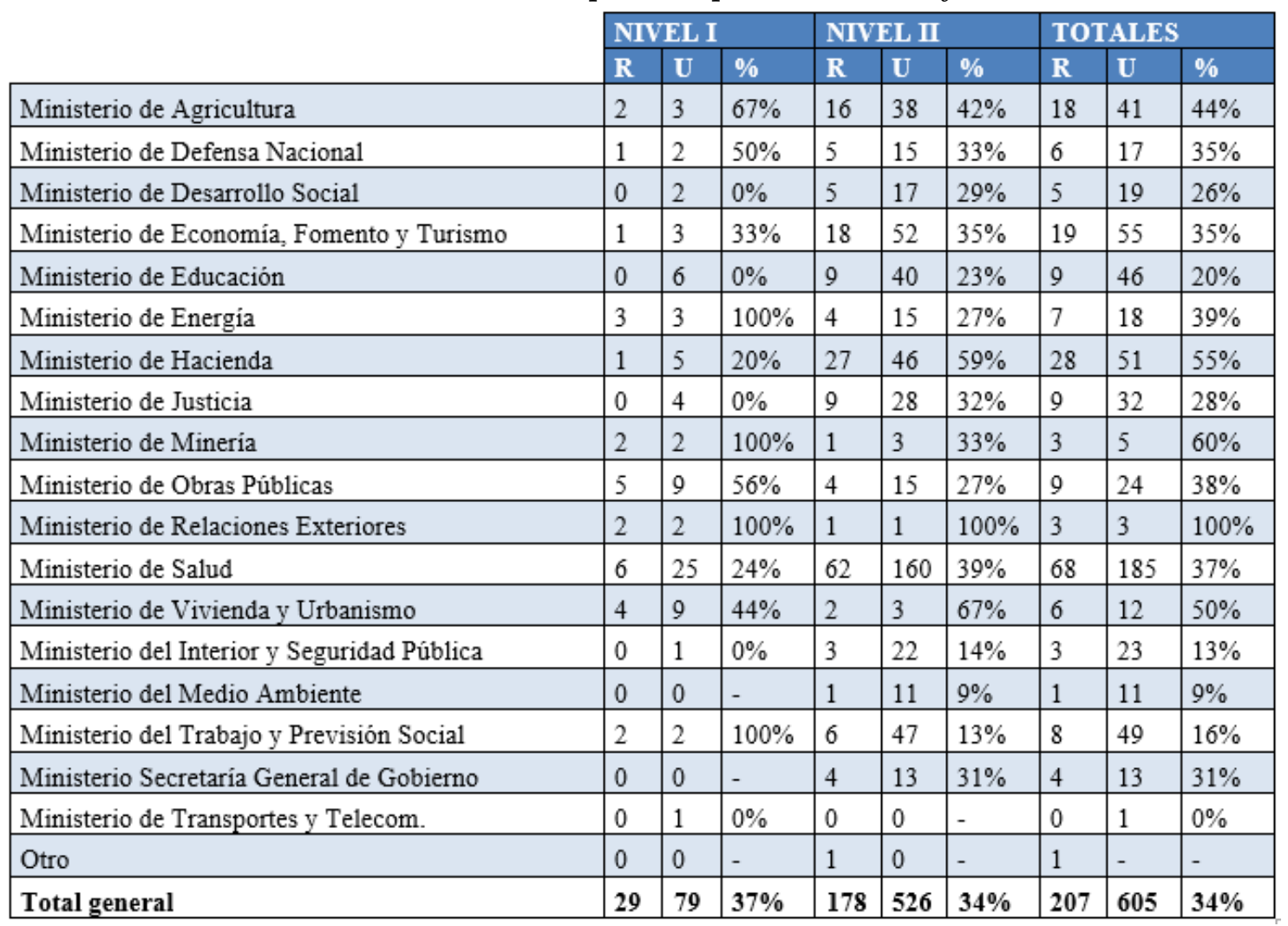

Fuente: Elaboración propia

peñaban, donde se podía marcar más de una alternativa, la mayoría de los directivos declararon pertenecer a entes ligados a la regulación y a la prestación de servicios sociales.

Otras funciones principales mencionadas de modo reiterado en la opción "Otro" del instrumento son de modo sintético y agrupado:

- Recaudación e inversión pública;

- Promoción de servicios sociales y de recreación;

- Protección y conservación de bienes públicos y de recursos naturales;
- Asesoría técnica y científica, investigación y desarrollo.

Asimismo se declararon en detalle la mayoría de los Servicios a los que pertenecían los encuestados, no obstante la pregunta directa era optativa. Si bien se puso como supuesto que los directivos podían dejar de contestar la encuesta si sentían que la encuesta buscaba identificarlos personalmente, se apreció que más del $80 \%$ declaró de modo directo o indirecto al Servicio al que pertenece.

Otro aspecto fundamental de la caracterización de la muestra es el promedio de permanencia en sus cargos. Los directivos públicos que contestaron la encuesta, han perdurado en sus cargos 2 años y 
Tabla 3: Gráfica de menciones a funciones principales de los Servicios

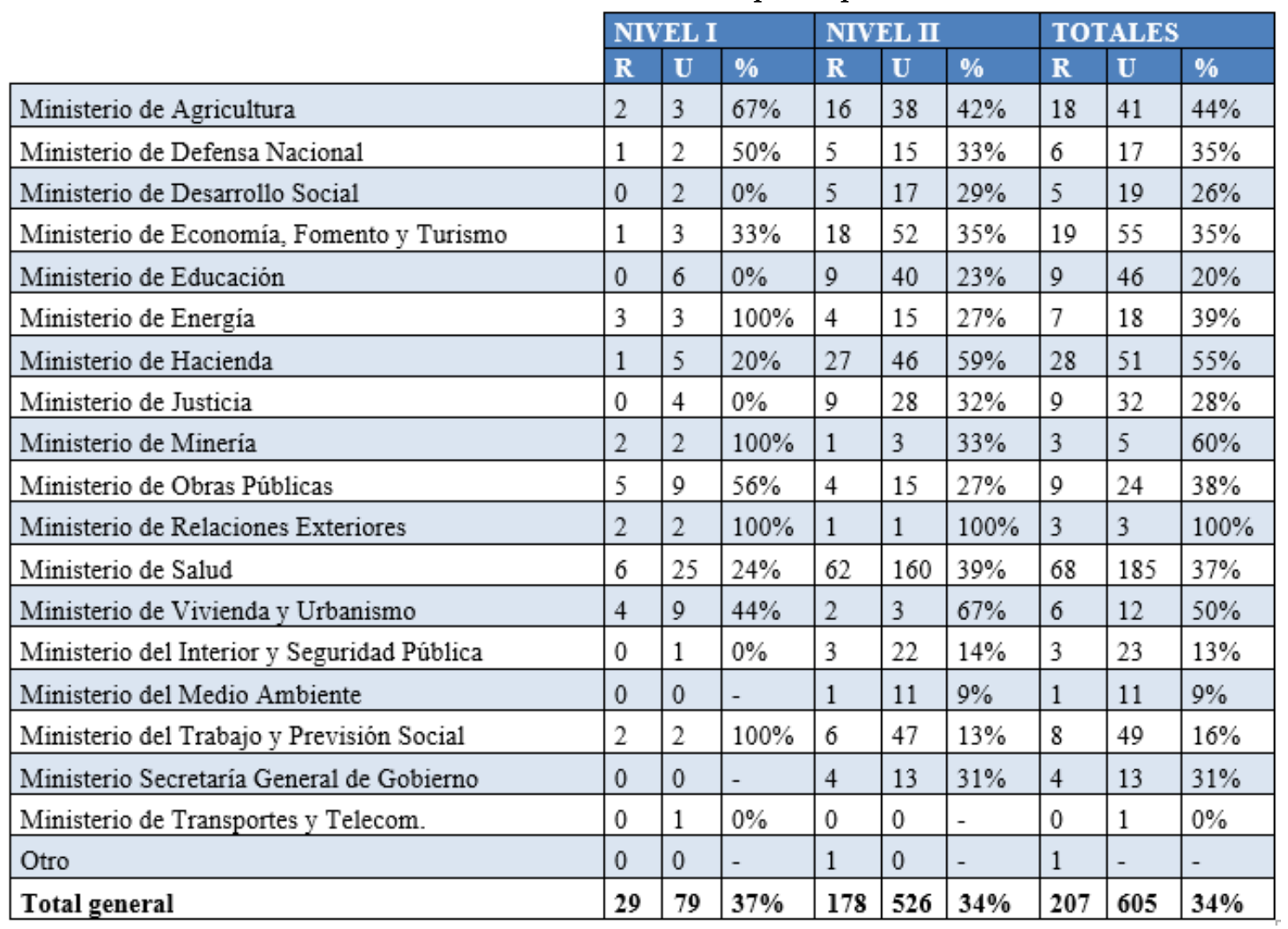

Fuente: Elaboración propia

6 meses, situación validada con profesionales de la Subdirección de Desarrollo de las Personas del Servicio Civil. En el caso del primer nivel jerárquico este promedio es de 2 años y 3 meses, y en cambio en el segundo nivel jerárquico este promedio aumenta a 2 años y 7 meses en el cargo. Es esperable que un cargo de primer nivel jerárquico dure en promedio menos que uno de segundo nivel, ya que el primero está más expuesto a susceptibilidades políticas y mediáticas que juegan en contra de la estabilidad del cargo. Cabe notar que una vacante desocupada en el Sistema puede ser subrogada por alguien al interior de la institución, o bien reemplazado por un Provisional y Transitorio designado por la autoridad correspondiente. Este último fenómeno se ha dado con mayor frecuencia en los cargos de primer nivel jerárquico.

El hecho de que el promedio de tiempo en el que desempeñan el actual cargo de los más de doscientos Altos Directivos Públicos encuestados sea menor a un período de ejercicio, que es de 3 años, habla de la juventud del Sistema y de las sensibilidades políticas asociadas aún a una cultura en desarrollo de la gerencia pública.

Dentro de la identificación a los encuestados, se pidió de forma libre que ingresaran hasta cinco títulos de pregrado y de postgrado que pudiesen tener, dejando como obligatorio al menos uno de ellos. Así se construyó la siguiente tabla considerando la principal profesión del encuestado según lo declarado. Se tomaron seis carreras como las más recurrentes, a partir de un reportaje del diario El Mercurio de diciembre de 2013, donde hace una infografía extensa de los ADP, con datos proporcionados por el Servicio Civil.

Se aprecia una fuerte predominancia de los encuestados en carreras del área de la administración y gestión, por sobre las cifras de El Mercurio. Además dentro de lo considerado como la opción "Otro", en la encuesta un $8 \%$ de las menciones se la llevaba la profesión de Ingeniero Agrónomo, vinculadas en general a los cargos del Ministerio de Agricultura, que fue uno de que tuvo gran representatividad en la muestra, por sobre la media de los Ministerios.

Otros antecedentes levantados sobre la formación de los directivos:

- 68 directivos declaran tener un magíster en áreas de administración y gestión.

- 33 directivos declaran contar con un magíster en otras áreas del conocimiento, gran parte en tópicos ligados al objeto de política de su ins- 
Tabla 4: Profesiones de los Altos Directivos Públicos

\begin{tabular}{|lll|}
\hline Profesiones & Encuesta & El Mercurio ${ }^{1}$ \\
\hline Ingeniería Comercial & $24 \%$ & $16 \%$ \\
\hline Ingeniería Civil y menciones & $23 \%{ }^{2}$ & $15 \%$ \\
\hline Medicina & $11 \%$ & $11 \%$ \\
\hline Derecho & $5 \%$ & $11 \%$ \\
\hline Contador Auditor y afines & $6 \%$ & $7 \%$ \\
\hline Administración Pública & $3 \%$ & $4 \%$ \\
\hline Otros & $28 \%$ & $36 \%$ \\
\hline
\end{tabular}

Fuente: Elaboración propia

titución.

- 5 directivos declaran tener un Doctorado (no médico).

- 70 directivos declaran tener entre 1 a 4 Diplomados de especialización en algunas materias.

- Algunos también declararon sus especialidades médicas.

Respecto al ámbito donde se desempeñaba el directivo, se les preguntó si durante los últimos 5 años anteriores a su nombramiento trabajaron en el sector público o privado. En este caso, la respuesta se condijo perfectamente a los datos que maneja el Servicio Civil, donde actualmente un $37 \%$ de los directivos seleccionados por el Sistema provienen del mundo privado. Aun así la cifra histórica de Altos Directivos Públicos que han sido nombrados vía Sistema es de $73 \%$ provenientes del sector público y un $27 \%$ restante del mundo privado. Cabe destacar que la brecha se aumenta levemente al referirse solamente al primer nivel jerárquico, donde en algunos casos los directivos han hecho carrera en el Servicio que se desempeñan, o bien su experiencia ha sido clave en el proceso de designación.

Se indagó también en el 63,8\% de los encuestados que se desempeñaba previamente en el Estado, si el cargo anterior que tenía también fue vía Sistema de Alta Dirección Pública y el 22,0 \% respondió afirmativamente, en su gran mayoría (93,1\%) en cargos de segundo nivel jerárquico. En promedio duraron en sus cargos 2 años y 9 meses aproximadamente. El hecho de que un quinto de los directivos encuestados haya declarado que el cargo anterior al actual en el que se desempeñó, fue vía Sistema de Alta Dirección Pública denota cierta validación y confianza en el sistema.

Para los 132 directivos que declararon haber trabajado en el Estado en los años previos, se indagó en el nivel jerárquico asociado a ese cargo, entendiendo que esta distinción no es clara en algunos Servicios públicos.

En contraste, se presenta lo declarado por aquellos que en los cinco años previos a su nombramiento actual, se desempeñaban en el sector privado u otra actividad no vinculada a la administración del Estado. Para ellos, la pregunta iba dirigida al cargo más alto desempeñado y se presentaba un set de posibilidades para que se asignaran a la que más le acomodase.

Dentro de la organización privada, académica u ONG a la que pertenecía anteriormente, ¿cuál es el nivel ejecutivo más alto que llegó a ocupar? (Marcar opción que más se asemeje)

Si bien no existe una adecuación de los sistemas jerárquicos público y privados, ya que ambos tienen sus complejidades y realidades organizacionales distintas, además de la gran heterogeneidad presentada en las mismas instituciones, se podría asociar el primer nivel jerárquico a las primeras dos clasificaciones anteriores, teniendo consideraciones de tamaño y volumen de lo administrado. A su vez una gerencia, podría representar algunos segundos niveles jerárquicos y lo propio un subgerente a un tercer nivel o jefe de división/departamento del sector público.

Distribución del Tiempo en relación a las Prácticas Directivas Uno de los objetivos específicos del estudio es poder identificar de modo cuantitativo, cómo usan el tiempo los directivos públicos, en el contexto de la cotidianidad de sus funciones. La gráfica devela una distribución normal esperable, que en promedio y a simple vista está por sobre una jornada laboral corriente de un funcionario público, establecida en el Estatuto Administrativo como de 44 horas cronológicas semanales.

Se nota una inclinación en la Ilustración 3 hacia más horas dedicadas a las funciones propias de 
Tabla 5: Indique el nivel jerárquico más alto desempeñado en la institución pública anterior

\begin{tabular}{|lllll|}
\hline Nivel & $\begin{array}{l}\text { Incentivos } \\
\text { económicos }\end{array}$ & $\begin{array}{l}\text { Reputación } \\
\text { profesional }\end{array}$ & $\begin{array}{l}\text { Experiencia } \\
\text { aprendizaje }\end{array}$ & $\begin{array}{l}\text { y Vocación } \\
\text { Servicio Público }\end{array}$ \\
\hline I & $17,2 \%$ & $23,3 \%$ & $24,6 \%$ & $34,8 \%$ \\
\hline II & $22,6 \%$ & $21,4 \%$ & $24,8 \%$ & $31,2 \%$ \\
\hline Total & $\mathbf{2 1 , 9} \%$ & $\mathbf{2 1 , 6 \%}$ & $\mathbf{2 4 , 8} \%$ & $\mathbf{3 1 , 7} \%$ \\
\hline
\end{tabular}

Fuente: Elaboración propia

Tabla 6: Nivel ejecutivo más alto desempeñado

\begin{tabular}{|lll|}
\hline Nivel ejecutivo más alto desempeñado & 9 & $12 \%$ \\
\hline Socio o duenio que administra la organización & 13 & $17 \%$ \\
\hline Gerente General / CEO/Rector / Preaidente / Director Ejecutivo & 29 & $39 \%$ \\
\hline Gerente de área / Director de area / Vicepresidente de area / Decano & 14 & $19 \%$ \\
\hline Subgerente de area / Subdirector de area / Vicedecano & 10 & $13 \%$ \\
\hline Otro & $\mathbf{7 5}$ & $\mathbf{1 0 0 \%}$ \\
\hline
\end{tabular}

Fuente: Elaboración propia

su cargo, lo cual era esperable. Notar también que en el gráfico agregado los funcionarios que declaran trabajar las 44 horas reglamentadas, corresponden íntegramente a directivos de segundo nivel jerárquico.

Respecto de la declaración de horas semanales que los directivos dedican a funciones y actividades propias de su cargo, es importante notar que en promedio significa más de un $20 \%$ de una jornada laboral normal del sector público. Inclusive un $18 \%$ de los encuestados declara dedicarle cerca del $40 \%$ extra más allá de la jornada normada, es decir más de 61 horas semanales.

Cabe señalar que no es habitual que un Alto Directivo Público haga uso de horas extraordinarias, las cuales están asociadas en su mayoría a funcionarios de niveles jerárquicos inferiores. Además internamente en las instituciones se entiende esta situación en la asignación especial en remuneración al Alto Directivo Público, por lo que políticamente sería mal visto hacer uso de ese beneficio, no obstante está más que evidenciado el tiempo "extra" que dedica un Alto Directivo Público a funciones propias de su cargo.

Mintzberg (2009b) asevera que "el trabajo del gerente incluye una preocupación perpetua: el gerente nunca puede estar libre para olvidar el trabajo, nunca tiene el placer de saber, aunque sea de modo temporal, que ya no queda nada pendiente por hacer" (26-27). Se entiende que un directivo, al menos asociado a un primer nivel jerárquico, ejer- ce el cargo no solo en el horario laboral, sino que también fuera de él, ya sea en actividades propias en horarios extraordinarios.

En relación al tema de las horas semanales dedicadas a la función propia del cargo, salta a la luz una encrucijada a la que se vio expuesto el Sistema. Es conocido en el SADP que los concursos que presentan mayor tasa de deserción son los del sector salud, en particular los asociados a los Servicios de Salud. Según la Memoria 2011-2012 del Consejo de Alta Dirección Pública, un 25,8\% se declaraban desiertos en el período considerado, es decir, no alcanzaban el mínimo de tres postulantes idóneos para conformar la nómina (Consejo de Alta Dirección Pública, 2013). Ante ello, la ley 20.498 promulgada en 2011, flexibilizó la dedicación exclusiva de los subdirectores médicos de los Servicios de Salud, quienes tienen un cargo de segundo nivel jerárquico. La modificación a la ley establece que los directivos podrán optar por una jornada de 44 horas semanales con dedicación exclusiva o bien de 33 horas semanales, no pudiendo ejercer un cargo de responsabilidad directiva en otro recinto de salud. Con ello se busca incentivar mayormente a los directivos a postular a los concursos, ya que en este sector es donde se aprecian las mayores brechas salariales en comparación al sector privado de salud.

Teniendo en consideración lo anterior, en la encuesta realizada, solo un $6,1 \%$ de los encuestados señaló dedicarle hasta 44 horas laborales a las funciones propias de su cargo. De ellos, la mitad corresponden a directivos de segundo nivel jerárquico de 
Figura 2: Nivel I ¿Cuántas horas cree usted que le dedica en una semana normal a actividades y funciones propias de su cargo (incluyendo fin de semana)?

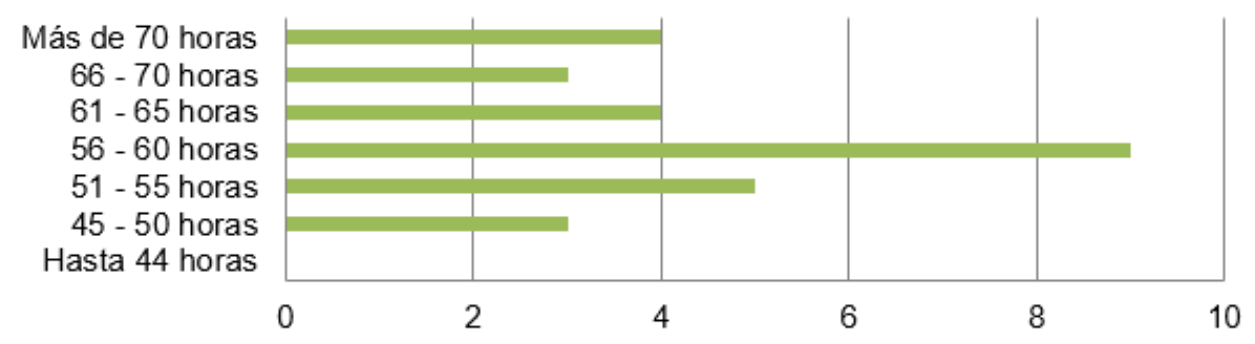

Fuente: Elaboración propia

Figura 3: Nivel II ¿Cuántas horas cree usted que le dedica en una semana normal a actividades y funciones propias de su cargo (incluyendo fin de semana)?

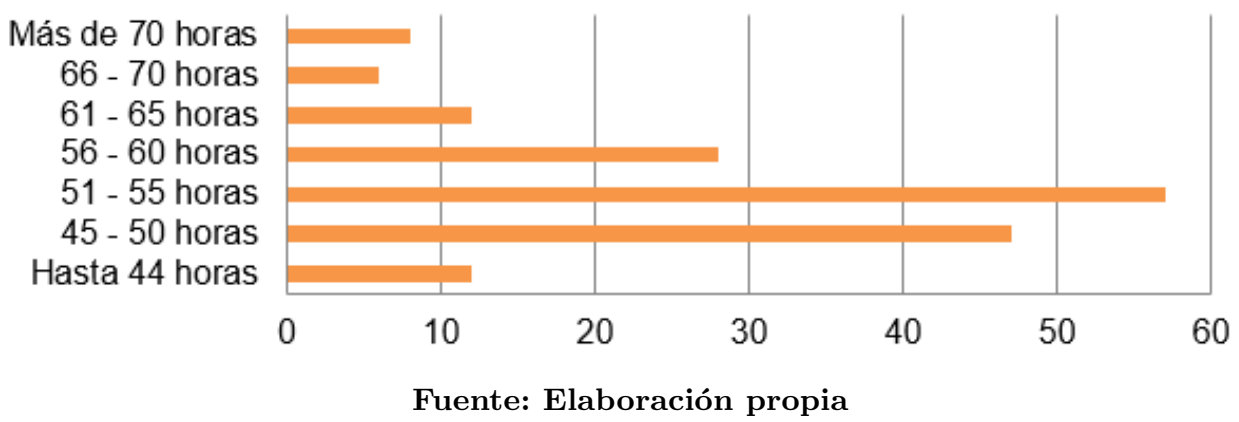

Servicios de Salud, lo que se podría interpretar que estarían haciendo uso del beneficio otorgado con la ley, ya que en general en el sector salud, se declara que en promedio las horas trabajadas es sobre las 55 horas y más aún, el $50 \%$ de los directivos encuestados que declaran trabajar más de 70 horas semanales (el rango máximo preguntado), provienen del sector salud.

La investigación del uso del tiempo de los directivos públicos se enfocó en una mirada principalmente exploratoria y descriptiva, donde no se buscaba encontrar un "mejor" uso del tiempo por sobre otro. Ahora bien, cuando una función se enmarca dentro de un contexto sí se podría catalogar como efectiva o no para la gestión del directivo.

Claramente con cerca de un $20 \%$ de su tiempo, los directivos los destinan a reunirse con su equipo cercano, sus asesores y equipos directivos. A su vez es la actividad que más directivos buscarían aumentar el tiempo que le dedican. La coordinación y tener al equipo alineado es parte fundamental del rol directivo.

La segunda actividad que más tiempo requiere aumentar en promedio, es la de leer documentos propios de sus funciones, esto se condice por lo explicitado en el acompañamiento directivo que se hizo en la etapa exploratoria, de prepararse previo a las reuniones y entender los temas sobre los que muchas veces tiene que tomar decisiones relevantes.

Dentro de las actividades a las que más se les dedica tiempo y deberían disminuirse según la mayoría de los encuestados, está el "Enviar y responder correos electrónicos" y el "Firmar y/o visar documentos".

Al desagregar la información por niveles, se observa que en el caso del Nivel I se acentúan de sobremanera las reuniones con internos y externos a la organización, y en ambos casos los directivos están conformes con el tiempo dedicado e incluso se podría aumentar. En el segundo nivel se mantiene el patrón del primer nivel, en relación a la evaluación de aumentar o disminuir los tiempos, donde por ejemplo el hecho de "redactar y corregir documentos" ocupa parte importante del tiempo de los directivos. A su vez se aumentado en relación al primer nivel la "gestión de la agenda personal" y la "mensajería instantánea".

A continuación, se presentan la distribución y evaluación cuantitativa de las funciones catalogadas como sustantivas. Es importante precisar que estas no compiten con las anteriores que tenían un marco de "operativas", seguramente muchas de las funciones sustantivas que desempeña un directivo en su quehacer, son a través de actividades operativas. 
Figura 4: Asumiendo como un $\mathbf{1 0 0 \%}$ el tiempo que le dedica a las siguientes $\mathbf{1 0}$ actividades operativas, ¿cómo distribuye su tiempo entre ellas?

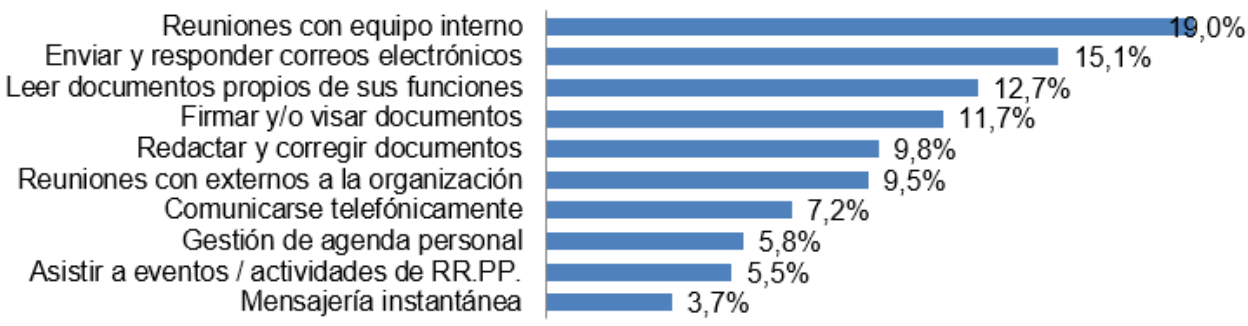

Fuente: Elaboración propia

Figura 5: Señale si usted cree que debería disminuir, mantener o aumentar, el tiempo que le dedica actualmente a las siguientes actividades operativas, de tal modo de aprovechar mejor su tiempo

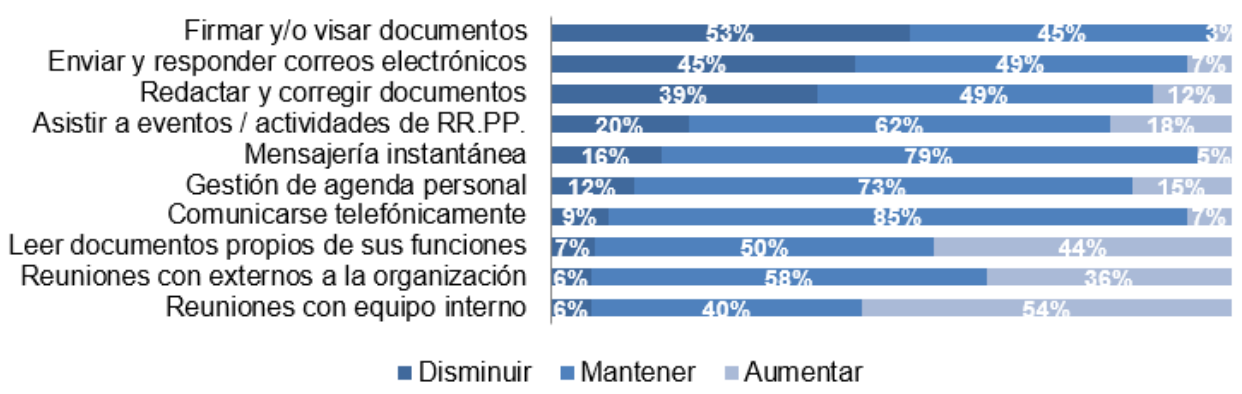

Fuente: Elaboración propia

Las funciones sustantivas requieren una evaluación más reflexiva y eso se expresa en que las varianzas son menores a la hora de responder. A todos los directivos les gustaría dedicarles más tiempo a muchas de ellas y seguramente con acciones que abarquen más de una, por ello la recomendación en la pregunta de considerar una aproximación razonable de diferenciación entre ellas para poder distribuir bien el tiempo.

En el primer nivel jerárquico, destaca por lejos la actividad a la que los directivos le dedican más tiempo: pensar estratégicamente. Esto es vital pues refuerza la ideal del Jefe de Servicio que debe conducir y pensar con antelación y decisión para dónde dirigir los rumbos y esfuerzos internos. En este nivel las decisiones que se toman pueden repercutir en más de 5.000 funcionarios de un Servicio de Salud, que atiende a más de 100.000 personas en sus centros de atención, por ejemplo. Redoblando la coherencia en este discurso, es declarada como la función que la mayoría cree que es a la que se debería aumentar más el tiempo.

A su vez existe una clara priorización con cuáles son las tres funciones más relevantes para los directivos de primer nivel jerárquico a las que no hay que disminuir el esfuerzo físico y mental en ningún sentido:
- Pensar estratégicamente

- Analizar información para la gestión

- Posicionar a la institución, negociar con externos, gestión política y gestión de redes

En contraste, una actividad en que la mayoría está por disminuir su dedicación es a la de ?apagar incendios? internos y negociar con los gremios. Seguramente esto desgasta mucho la labor diaria y se producen muchos eventos distintos, que en ocasiones no tienen relación y por ende no hay un denominador para su solución.

Llama la atención que en el segundo nivel destaque el hecho sustantivo de liderar equipos, por sobre el primer nivel, que está mayormente abocado a el pensamiento estratégico de la organización y al constante análisis e información para poder gestionarla mejor.

En el nivel II, baja considerablemente la "gestión de prensa y vocería", el posicionamiento institucional y la gestión política, lo que tiene que ver con que en muchos casos están en la segunda línea de la organización, aunque no en todos. En contraste, aumentan el liderazgo de equipos y la gestión interna del presupuesto, es decir, la administración de los recursos. 
Figura 6: Nivel I, Asumiendo como un $\mathbf{1 0 0 \%}$ el tiempo que le dedica a las siguientes 10 funciones sustantivas, ¿cómo distribuye su tiempo, considerando una aproximación razonable de diferenciación entre ellas?

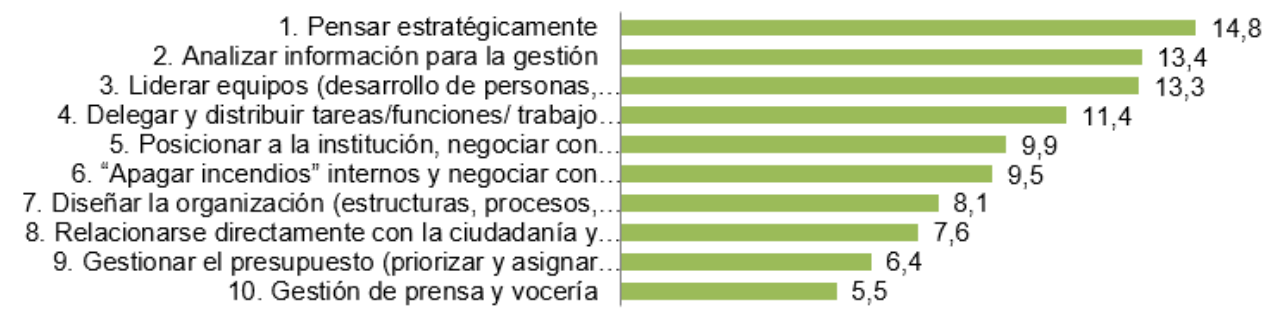

Fuente: Elaboración propia

Figura 7: Nivel I, Señale si usted cree que debería disminuir, mantener o aumentar, el tiempo que le dedica actualmente a las siguientes funciones sustantivas, con el fin de agregar más valor a su organización

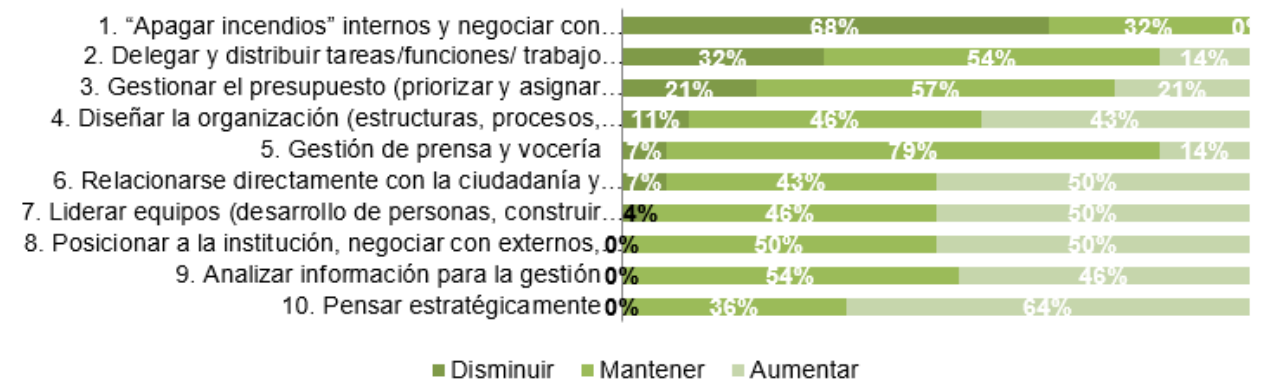

Fuente: Elaboración propia

Figura 8: Nivel II, Asumiendo como un $100 \%$ el tiempo que le dedica a las siguientes 10 funciones sustantivas, ¿cómo distribuye su tiempo, considerando una aproximación razonable de diferenciación entre ellas?

1. Liderar equipos (desarrollo de personas,...

2. Analizar información para la gestión 3. Pensar estratégicamente 4. "Apagar incendios" internos y negociar con

5. Diseñar la organización (estructuras,...

6. Delegar y distribuir tareas/funciones/ trabajo

7. Gestionar el presupuesto (priorizar y asignar.

8. Posicionar a la institución, negociar con.

9. Relacionarse directamente con la.

10. Gestión de prensa y vocería

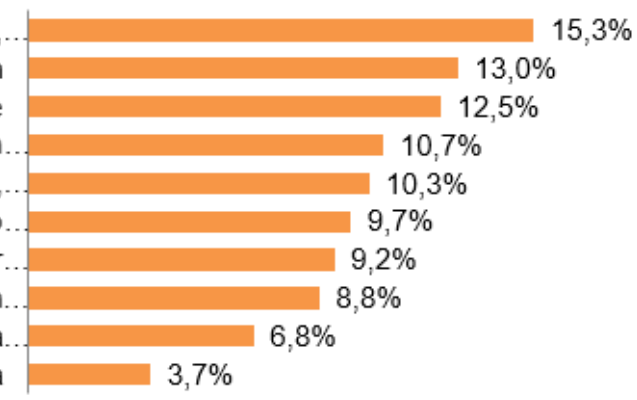

Fuente: Elaboración propia 
Figura 9: Nivel II, Señale si usted cree que debería disminuir, mantener o aumentar, el tiempo que le dedica actualmente a las siguientes funciones sustantivas, con el fin de agregar más valor a su organización

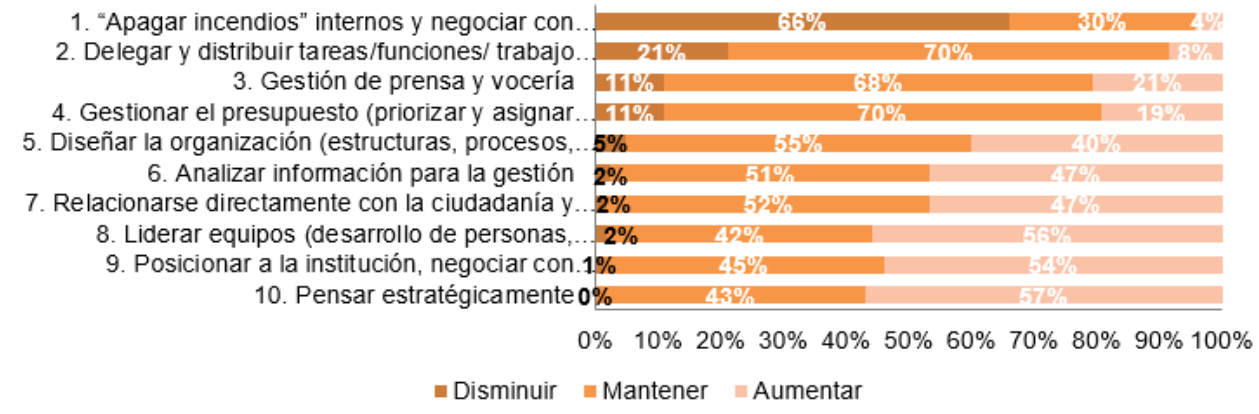

Fuente: Elaboración propia

En general la relación con la ciudadanía y los usuarios es baja en tiempo y a un $47 \%$ le gustaría aumentar esa cifra. Probablemente las contingencias impiden la dedicación a la gran parte de los Altos Directivos Públicos les gustaría tener.

\section{Identificación de Competencias de los Altos Directivos Públicos}

La segunda parte de la encuesta tenía relación con las capacidades y atributos de los Altos Directivos Públicos. En una primera etapa se buscó conocer cuáles son las competencias personales para ejercer la gerencia, para luego pasar a las competencias interpersonales, todas estas inspiradas en Mintzberg (2009b) y su clasificación de competencias de los gerentes y en los planos que se desarrollan. Una última parte, ligada a las competencias relativas al sector público chileno con sus particularidades. Al igual que la parte de uso del tiempo, se muestran en primer lugar los resultados agregados para luego pasar a observar las diferencias existentes entre los distintos niveles jerárquicos.

Es importante notar que la escala Likert utilizada con 5 clasificaciones fue agrupada de un modo distinto para el análisis de las gráficas que se muestran a continuación en este subtítulo. Existe un sesgo de los directivos al autoevaluar sus competencias, al no contar con la información de contraste pertinente, por ejemplo de su equipo cercano y tal vez, la autoridad a la cual reportan. Por tanto, se decidió agrupar las 3 primeras opciones de la escala que correspondían a "no desarrollada", "poco desarrollada" y "medianamente desarrollada", en una que se presenta en las gráficas como "medianamente o menor".

No obstante esta decisión tiene asidero en que estos directivos ya han pasado arduos procesos de selección con cientos de competidores y con empresas de head-hunters que han certificado sus competencias para ciertos ámbitos, así como también instancias frente a la institucionalidad correspondiente en su selección como el Consejo de Alta Dirección Pública en el caso de los directivos de Nivel I. Por tanto es razonable esperar que al menos ellos no se califiquen en varias de las categorías alguna de las primeras dos opciones de la escala.

En el desarrollo de competencias personales los directivos se evalúan en general bastante bien y no hay mayores diferencias entre lo planteado por los directivos nivel I y nivel II. Salvo en "programar la agenda", donde se evidencia la mayor diferencia en relación a las otras y un $42 \%$ declara tenerla medianamente desarrollada o menor a esto. Esto se podría explicar por las contingencias que ocurren en el día a día y la incapacidad de anteponerse a ellas, lo que repercute en la programación de la agenda.

En una segunda etapa de evaluación aparecen las competencias interpersonales. En primer lugar aquellas que tiene relación con liderar personas y equipos de trabajo.

En el marco de Mintzberg (2009b) estas se separarían como: a) Liderar a las personas (seleccionar, enseñar/servir de mentor/entrenar, inspirar); y b) Liderar grupos (resolver y mediar conflictos, armar equipos, dirigir reuniones)

Se devela que la competencia de "seleccionar personas" es aquella donde los Altos Directivos Públicos se sientes más débiles. También al menos un cuarto de los encuestado se declara medianamente conforme con su performance en "enseñar/servir de mentor/entrenar", competencia clave a la hora de ser un "buen jefe" para los funcionarios públicos.

En contraste, en "dirigir reuniones" es donde los directivos se sienten más seguros frente a grupos de trabajo. En el caso de los directivos nivel I, los 
Figura 10: Nivel I, ¿Con qué nivel de profundidad cree que tiene desarrolladas las siguientes capacidades y atributos personales?

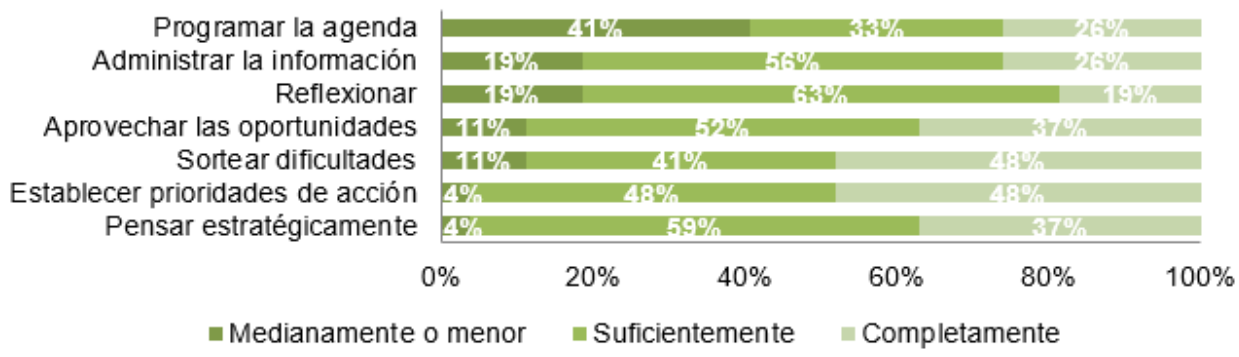

Fuente: Elaboración propia

Figura 11: Nivel II, ¿Con qué nivel de profundidad cree que tiene desarrolladas las siguientes capacidades y atributos personales?

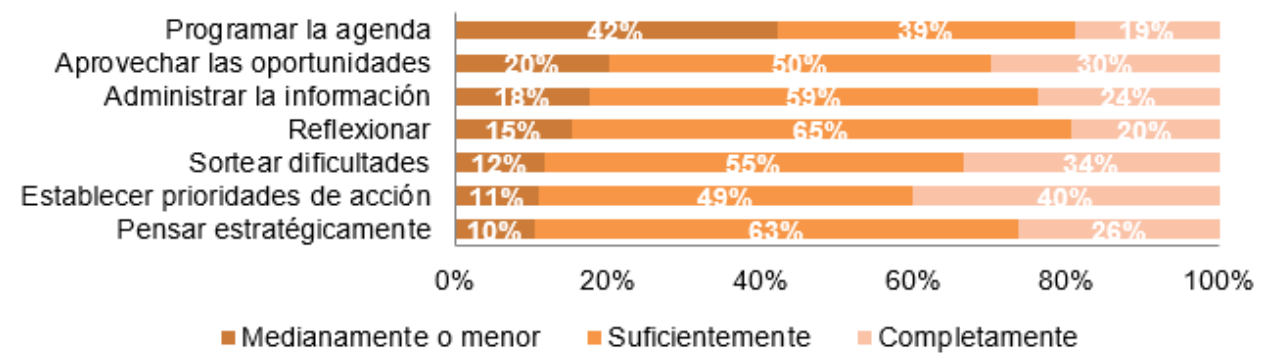

Fuente: Elaboración propia

Figura 12: Nivel I, ¿En qué nivel considera que tiene desarrolladas las siguientes capacidades y atributos interpersonales, respecto a liderar personas y grupos de trabajo?

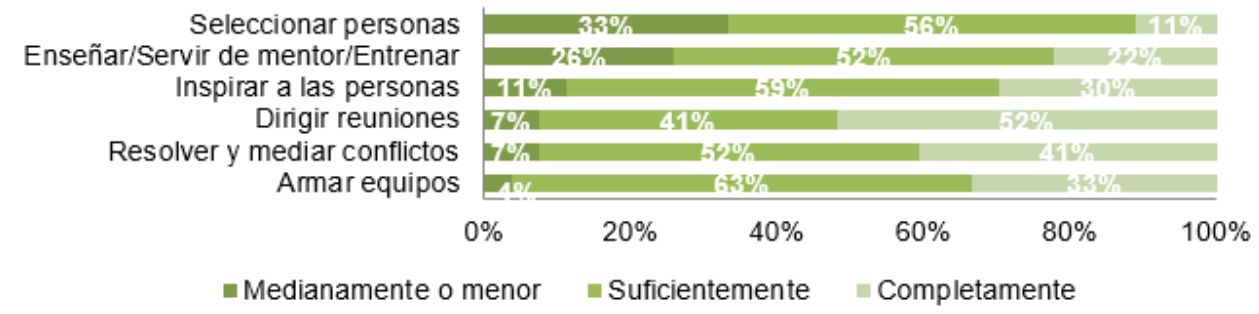

Fuente: Elaboración propia

Figura 13: Nivel II, ¿En qué nivel considera que tiene desarrolladas las siguientes capacidades y atributos interpersonales, respecto a liderar personas y grupos de trabajo?

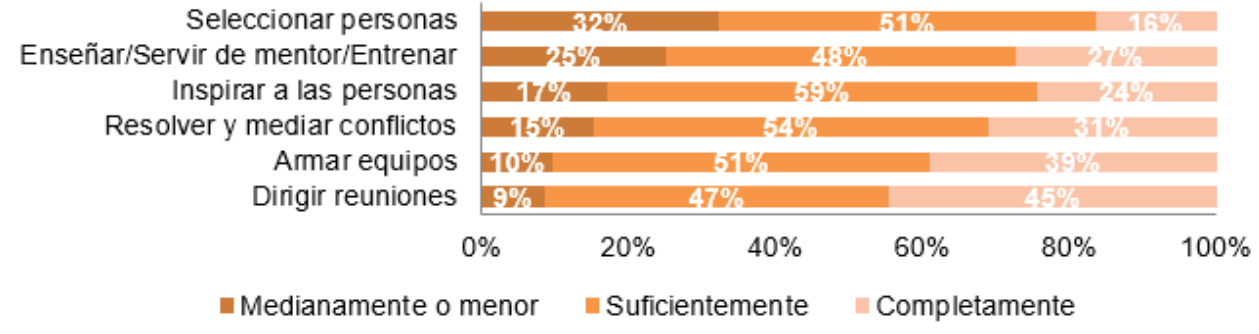

Fuente: Elaboración propia 
que creen que tienen la competencia completamente desarrollada, llega a $52 \%$.

Por otra parte están aquellas competencias interpersonales que están definidas como la capacidad de los directivos para relacionar su organización en el caso del nivel I y algunos del nivel II, y para relacionar su unidad en el caso de los de nivel II.

En ese sentido se diferencian en: a) Liderar la organización/la unidad; y b) Relacionar la organización/la unidad. En este estudio se tomó solo la segunda acepción para encuestar a los directivos.

En el caso de las competencias interpersonales para con el entorno, hay diferencias importantes entre el nivel I y el II, tanto en representar y relaciones públicas, gestión de redes, y en generar alianzas y prácticas colaborativas. Evidentemente este es un punto en el que los directivos de segundo nivel debiesen estar mayormente reforzados.

A su vez se observa que aparece la variable "no aplica" como una de las posibles respuestas por parte de los directivos.

Finalmente en esta etapa, aparecen las competencias que debe tener un directivo en relación a sus capacidades para lidiar con el sector público chileno, con sus complejidades y desafíos. Esta es la única de este set de preguntas que no proviene de la literatura.

En promedio, es acá donde los directivos se encuentran menos preparados. En particular en los primeros dos puntos, son aquellos que salieron más destacados en el focus group de Altos Directivos Públicos, como temas con los cuales tenían que lidiar en la cotidianeidad y muchas veces se pierden desde su aproximación más técnica al sector público.

Otro aspecto interesante es que aquellas competencias más estándares relativas al sector público, como rendir cuentas formales o la gestión del presupuesto, están relativamente mejor desarrolladas que el resto, lo que supone un aprendizaje del funcionamiento del sistema y su relación a través del cargo que ocupa para lograr cumplir con lo necesitado según sea el caso.

Queda claro que aún hay un importante desafío en gestión política de los directivos para con sus stakeholders. Este es uno de los aspectos que hace distinto a los directivos públicos en cuanto a su mandante en relación al sector privado, entre otras cosas.

En este cuarto set de competencias es donde se hace notar una mayor diferencia entre lo declarado por parte de los distintos niveles jerárquicos. El nivel I aparece con capacidades y habilidades más desarrolladas para enfrentarse a las situaciones que se gatillan a partir de lo enunciado en la gráfica. A su vez abre una serie de interrogantes relativos al perfil del directivo, la complejidad de entender el entorno y el alcance del rol técnico en un nivel II.

Además la opción "no aplica" cobra gran relevancia en el segundo nivel jerárquico, donde se da una fuerte señal que no se han visto enfrentados a situaciones en las cuales necesiten contar con las competencias listadas. Esto podría ser una preocupación en un sentido de proyecciones.

Por último, se realizó una pregunta inspirada en una presentación de la Consejera María Loreto Lira (2013), del Consejo de Alta Dirección Pública, donde cuestiona los incentivos y motivaciones que tiene una persona para desempeñarse como directivo público con todo lo que ello conlleva, lo que muchas veces implica gran costo personal en distintas dimensiones.

Llama la atención la alta valoración que se hace de la experiencia y aprendizaje que se gana con ser directivo público, por sobre la reputación profesional. En cuanto a los incentivos económicos, se explica que en el primer nivel sean menos valorados, ya que dentro del mercado laboral el nivel al que ellos podrían aspirar en el sector privado podría ser bastante mayor. En el caso de los segundo nivel esa competencia entre público-privado, es menor sobretodo dependiendo del sector económico en el que se mida.

\section{Focus Group a Altos Directivos Públi- cos Nivel I}

Un método efectivo y directo para conocer la impresión de primera fuente sobre estos temas, es un grupo focal. Se invitó en una primera instancia a aquellos directivos del Primer Nivel Jerárquico que contestaron afirmativamente en la encuesta, que les gustaría ser contactados para ahondar su aporte a esta investigación. Posterior a la respuesta de este primer grupo, se envió una invitación masiva con la que se completó el número de asistentes.

Los asistentes a la actividad fueron Magdalena Krebs, Francisco Echeverría, Valentín Díaz, Patricia Jaime, María Isabel Castillo, Jaime Binder y María Angélita Silva.

Para poder analizar de mejor modo lo expresado por los asistentes en este taller, se diseñaron dimensiones y subdimensiones de análisis. Estas tomaron como base lo realizado en la encuesta y se agregaron otras dimensiones relevantes para los objetivos 
Figura 14: Nivel I, Según su ámbito de autoridad, ¿en qué nivel considera que tiene desarrolladas las siguientes capacidades y atributos interpersonales, para relacionar su organización/unidad con el entorno?

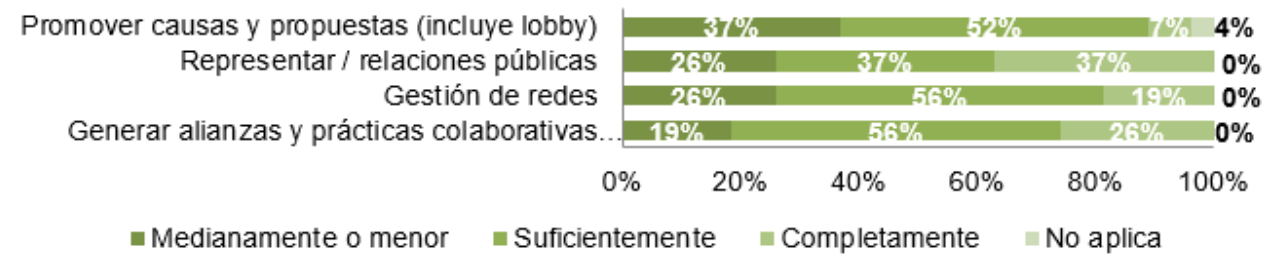

Fuente: Elaboración propia

Figura 15: Nivel II, Según su ámbito de autoridad, ¿en qué nivel considera que tiene desarrolladas las siguientes capacidades y atributos interpersonales, para relacionar su organización/unidad con el entorno?

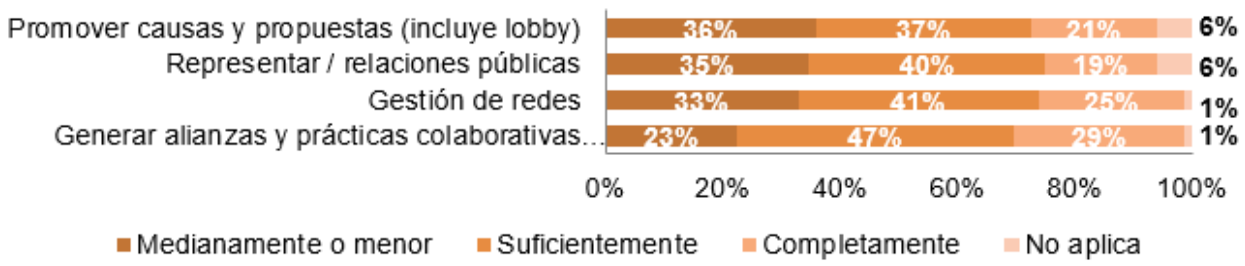

Fuente: Elaboración propia

Figura 16: Nivel I, ¿En qué nivel considera que tiene desarrolladas las siguientes capacidades y atributos, relacionadas al sector público?

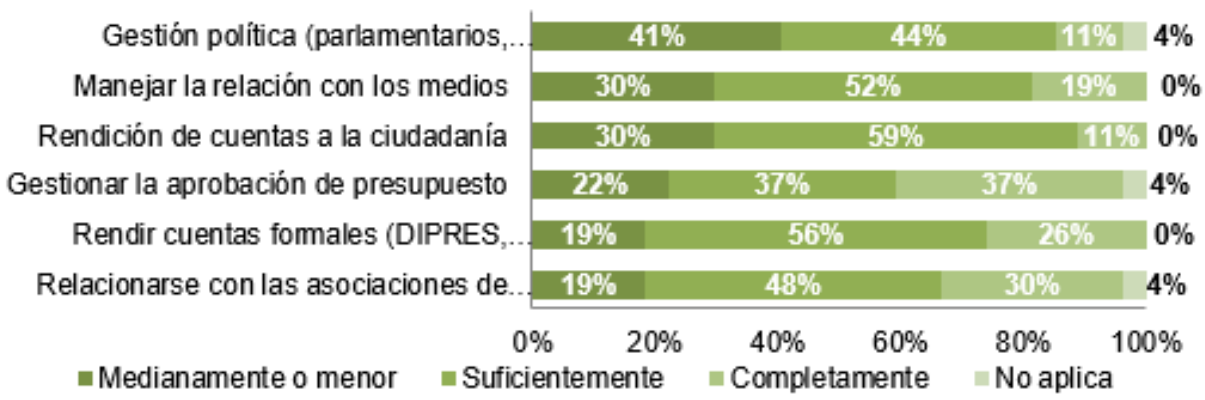

Fuente: Elaboración propia

Figura 17: Nivel II, ¿En qué nivel considera que tiene desarrolladas las siguientes capacidades y atributos, relacionadas al sector público?

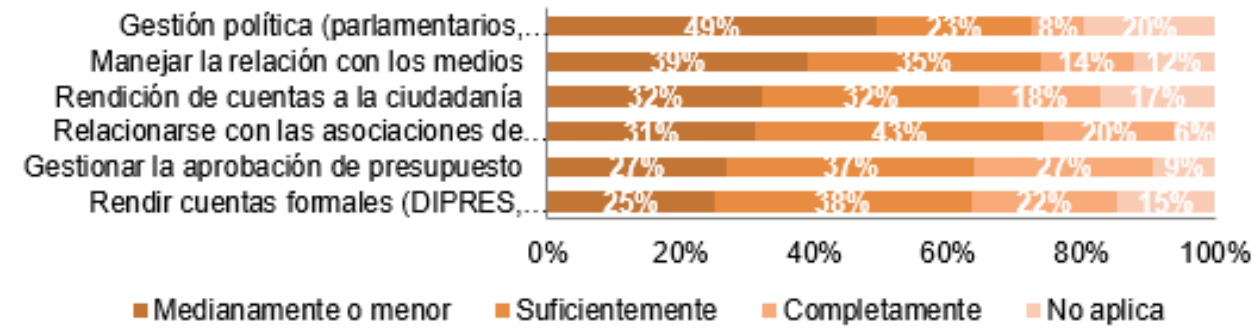

Fuente: Elaboración propia 
Tabla 7: Finalmente, entendiendo la motivación personal como un elemento clave para un desempeño adecuado de la práctica gerencial ¿diga usted cómo distribuiría las siguientes motivaciones personales en su caso?

\begin{tabular}{|lllll|}
\hline Nivel & $\begin{array}{l}\text { Incentivos } \\
\text { económicos }\end{array}$ & $\begin{array}{l}\text { Reputación } \\
\text { profesional }\end{array}$ & $\begin{array}{l}\text { Experiencia } \\
\text { aprendizaje }\end{array}$ & $\begin{array}{c}\mathbf{y} \begin{array}{l}\text { Vocación } \\
\text { Servicio Público }\end{array} \\
\text { I }\end{array}$ \\
\hline II & $22,2 \%$ & $23,3 \%$ & $24,6 \%$ & $34,8 \%$ \\
\hline Total & $\mathbf{2 1 , 9 \%}$ & $21,4 \%$ & $24,8 \%$ & $31,2 \%$ \\
\hline
\end{tabular}

Fuente: Elaboración propia

del estudio y de las preguntas de investigación.

\section{Efectividad de un Directivo}

La gestión efectiva de una organización requiere de un conjunto de elementos como liderazgo, recursos, incentivos, libertad de gestión y valores congruentes. Todo esto es compatible con el enfoque de estudio de las prácticas directivas que posibilitan la efectividad de la gestión o bien la hacen más probable.

En ese contexto, hubo mención a ciertas prácticas que los altos directivos ven como indispensables para ser efectivos en su cargo. Una de las más nombradas tiene que ver con estar rodeado de un equipo de calidad y confianza, que les permita tener espacio para dedicarle a cosas más sustantivas del cargo como tal. Por otra parte, también se menciona la importancia de una buena relación con dicho equipo.

Otro recurso nombrado, fue el del uso de la tecnología, mediante el cual también habría un ahorro de tiempo, el cual debiera ser utilizado en una de las tareas más relevantes para los altos directivos, como lo es, tomar decisiones.

La claridad de propósitos y objetivos del cargo, también es mencionado como un punto importante a la hora de ser efectivo como alto directivo.

Un punto muy interesante que cruzó el tema de la gestión efectiva, tuvo que ver con la discusión que se sostuvo sobre el rol técnico versus un rol político que tiene que tener un Alto Directivo Público. Mientras algunos mencionaban la importancia de que su rol sea exclusivamente técnico, otra voz menciona que es imposible mantenerse en el sistema público sin tener algún conocimiento político.

\section{Necesidad de Sistemas de apoyo}

Un comentario compartido de los directivos, es la necesidad de apoyo tanto en el proceso de inducción como durante el ejercicio de su cargo. En esta labor de apoyo, se menciona, en algunos casos, al Servicio Civil, como un organismo que está pendiente de sus necesidades y con una constante preocupación por los problemas que vayan surgiendo.

Si bien se declaran que existen las instancias propiciadas por el Servicio Civil para compartir entre directivos, la limitante del tiempo salta a la luz, tal como se evidenció con los datos cuantitativos de la encuesta, lo que dificulta asistir a estas reuniones.

Se reconoce que el Servicio Civil proporciona instancias de convivencia y también otorga capacitaciones, valorando su labor. Sin embargo, también se contrasta con el hecho de que hay una falencia en este organismo respecto al acompañamiento en el proceso de inducción.

Existe un sentimiento generalizado de "soledad en el cargo", por el hecho de no poder compartir sus problemas con otras personas. Para este problema, se manifiesta la importancia de instancias para compartir experiencias, y buscar redes de apoyo en otros Altos Directivos Públicos, con el fin de sentirse acompañados y apoyados en los problemas comunes que surgen en sus cargos.

En el año 2010 existió un programa de mentoring encabezado por el Servicio Civil, el cual no perduró en el tiempo, al parecer debido a motivos económicos. Dado lo mencionado por los directivos, pareciera ser relevante que se retomaran este tipo de iniciativas por parte del Servicio Civil.

Por otra parte, en algunos casos, el principal apoyo es el que les otorgan los Ministros o Subsecretarios (dependiendo del Servicio). Sin embargo, también se menciona que debido a que en algunos casos, el Ministro o Subsecretario pueden ir rotando, depende de la disposición de éstos, el apoyo que les otorguen a los directivos. 
Tiempos de instalación en el cargo directivo

En general, el tiempo que los directivos estiman que necesitan para instalarse en el cargo es de tres a seis meses. Sin embargo, la mayoría menciona que existe una diferencia importante en el tiempo de inducción, dependiendo de si se viene desde "afuera" o desde "adentro". Estas categorías se refieren principalmente a si se viene desde el mundo privado o desde un cargo público anterior, aunque también atañen a si se viene del mismo Ministerio o Servicio o desde otro sector dentro de la administración pública.

Además de estas distinciones, la particularidad del cargo de Alto Directivo Público, de todas formas hace necesario un tiempo de aprendizaje en este cargo, ya que aunque se venga del sector público y se conozcan sus limitaciones y virtudes, tener ese cargo en particular requiere cierto período de adaptación.

Un ejemplo que llamó la atención en relación a fortalecer competencias para enfrentarse al período de instalación en el cargo, fue la contratación de un servicio de coaching para el desarrollo de habilidades necesarias para un cargo de estas características.

\section{Heterogeneidad de las instituciones}

Los Altos Directivos Públicos consideraban importante la diferencia que hay entre un Servicio y otro. Estas diferencias, se pueden ver en el ámbito de las vocerías, la relación con la ciudadanía y las relaciones con otros organismos. En cuanto a las explicaciones de estas diferencias, se encuentra la misión u objeto de política del Servicio en sí, pero también hay factores ministeriales que pueden hacer la diferencia. El rol de la autoridad política es un factor de incidencia, en este caso, para respetar la heterogeneidad de los Servicios bajo ellos.

\section{Competencias Personales}

El pensamiento estratégico es un punto fundamental en la tarea que los atañe como Altos Directivos Públicos, y se destaca que pensar estratégicamente es un requerimiento para ser un interlocutor efectivo entre el interior y exterior de la organización en sí. Además, es necesario contar con un espacio temporal, como condición, para poder desarrollar esta competencia.

Respecto a las dificultades que puede presentar el cargo, se presenta nuevamente como fundamental el tener un equipo de confianza, para poder dedicarle tiempo a poder lidiar con ciertos imprevistos y "apagar los incendios" que se van sucediendo en la contingencia institucional.

Como Alto Directivo hay que saber administrar los tiempos, que son distintos a los tiempos privados, ya que éstos últimos son mucho más rápidos. Un Alto Directivo, tiene que saber aprovechar ese tiempo entre que las decisiones se toman y se ejecu$\tan$ (con todas las restricciones que tiene el mundo de la administración pública), y usarlo con el fin de abocarse de otros temas dentro de sus tareas. El saber ocupar el tiempo de manera óptima, dentro de un marco legal, se esboza como importante para cumplir una función eficiente y saber aprovechar las oportunidades del entorno.

\section{Competencias Interpersonales respecto a li- derar}

Como ya se ha mencionado, el tener equipos capacitados y de confianza es de vital importancia para los directivos públicos. Es por eso, que sus capacidades respecto a crear buenos equipos incide significativamente en su desempeño general. Para lograr tener buenos equipos hay que seleccionar a quienes tienen más habilidades y saber escoger a determinadas personas de confianza y con competencias específicas para las necesidades que ellos presentan.

Dar autonomía al equipo es fundamental, en cuanto esto permite dedicarle tiempo a temas sustantivos, y solamente revisar o dirigir las tareas de estos equipos, pero con la seguridad que están haciendo bien su trabajo.

\section{Competencias Interpersonales para relacio- nar a la organización}

La comunicación con el entorno debe ser de invitación, para que ocupen sus servicios, por ende, el directivo debe tener la capacidad de generar una comunicación atractiva. En un segundo caso, la relación con el entorno, también por la particularidad del servicio, tiene una dificultad distinta, ya que en los temas que le atañen, es difícil que no haya críticas, incluso cuando se quiere invitar a la ciudadanía a cosas positivas. Por tanto, es fundamental generar alianzas y prácticas colaborativas con el entorno.

En general, las competencias de promoción de ciertas causas o apoyos en instancias políticas, incluyendo el rol del lobby, es un aspecto que le falta desarrollar a los directivos que cumplen un rol más técnico, prefiriendo delegar la responsabilidad en el 
Subsecretario o Ministro según corresponda. Este es un aspecto a mirar más de fondo en el sentido de empoderamiento político que debe tener un Alto Directivo Público, independiente de que su Servicio cumpla un rol técnico.

Un tema que surge con fuerza es la autonomía que los directivos necesitan tener respecto a las autoridades. Esta autonomía es planteada fundamentalmente en el sentido de no quitarles tiempo a sus superiores, ya sea el Subsecretario o el Ministro, para que puedan realizar otro tipo de labores que les demanda el cargo. La autonomía, por lo tanto, es vista como una competencia necesaria en los Altos Directivos Públicos, y es parte de hacer bien su trabajo, y estar empoderados en su cargo, como para no tener que depender de sus jefes a cada momento.

En esta misma línea, lo importante es llamar a sus superiores, sólo en situaciones que lo ameriten, que tengan que ver con la contingencia o con decisiones de importancia mayor.

\section{Competencias relacionadas al sector público}

Al momento de relacionarse con funcionarios o asociaciones de funcionarios, sí se mencionan ciertas competencias o estrategias que deben desplegar los directivos para poder tener un mejor diálogo. Por un lado, un ambiente grato y de cercanía se esboza como algo muy útil, y en este mismo sentido, el que los funcionarios - gracias a las habilidades que tenga el director- entiendan las condiciones objetivas del Servicio, y al mismo tiempo, tengan la confianza en que sus peticiones serán tomadas en cuenta, es vislumbrado como algo de suma importancia al momento de mantener buenas relaciones. Tras ello se evidencia una clara práctica directiva, en la forma de hacer las cosas.

Es importante que el director cree estrategias para poder lidiar con estas asociaciones, sin que pierdan demasiado tiempo en ello, y exista un orden y un conocimiento previo, para poder hacer más efectiva la relación con los funcionarios. En estos casos la estrategia consiste en tener un encargado que se dedique exclusivamente a esto, y que logre filtrar los casos y que si existe una necesidad de reunirse con funcionarios o asociaciones de funcionarios, los temas estén ordenados y revisados con anterioridad.

En el caso del manejo de la relación con los medios, es considerado por los directivos como un asunto complejo con el que deben lidiar en el ejercicio de su cargo, aunque el tema de las vocerías tenga un papel diferenciado dependiendo del Servicio específico o el Ministerio al que pertenece dicho Servicio. Dentro de las competencias necesarias pa- ra realizar una buena interacción con la prensa, se nombran algunas habilidades adicionales a las propias de un directivo, como tener un tono adecuado, saber qué cosas conviene decir y qué no. Otro elemento necesario que se esboza, es tener un equipo comunicacional, en algunos casos, que pueda facilitar la tarea del directivo.

También aparece como necesidad de tener una buena vocería y gestión de prensa, el tener una cierta preparación previa, como información preparada y ciertas ideas fuerza que transmitir. Por otra parte, se plantea que exponerse a la prensa sólo en momentos de defensa tiene un alto riesgo para el directivo. Además en la actualidad, el tema se vuelve aún más complejo y requiere de mayores competencias comunicacionales, por el "ánimo", generalizado de crítica hacia la gestión gubernamental, tanto por parte de la ciudadanía como de los medios de comunicación.

En relación a la participación ciudadana, en algunos directivos aparece la necesidad de que ésta se aparezca en ciertos contextos específicos, y no como parte de sus tareas diarias, ya que es necesario para ellos tener un espacio donde puedan tomar decisiones sin esa presión. Para eso, se ve como necesario desactivar parte de la participación ciudadana

La temporalidad en el cargo y el sentir dentro de la institución que se "está de paso", implica dificultades para ejercer una buena labor como directivo, y también los problemas que esto puede traer a los Servicios en su funcionamiento. Para solucionar los problemas que crea en la institución que se dé esa práctica, una de las directivas señala que su estrategia ha sido la de institucionalizar sus decisiones, con el fin de no generar una inestabilidad dentro de la organización cada vez que haya cambio de Altos Directivos. Al mismo tiempo, se menciona que debe existir un mecanismo que permita que la permanencia en los cargos no sea tan inestable.

\section{Uso del tiempo en funciones operativas}

1. Firmar y/o visar documentos En cuanto a la revisión de documentos, y firma de ellos, dos de los directivos, señalan que este menester les ocupa un par de horas al día, lo que desde la óptica de la eficiencia es muy caro para la gestión del Estado. El uso de esas horas, es considerado parte de su "negocio", ya que significa la aprobación o reprobación de ciertas tareas que han delegado.

Existen tareas operativas que los directivos no ven de buena forma, ya que sienten que le quitan tiempo a sus labores sustantivas. Por ejemplo, las compras y licitaciones, se ven como te- 
mas que obstaculizan el ejercer de mejor manera su cargo.

2. Enviar y responder correos electrónicos El tiempo en enviar y responder correos electrónicos, si bien no es mencionado en todos los casos, es parte constitutiva de su rol como directivo, en cuanto a que mediante esta herramienta, ellos pueden revisar documentos, ejercer tareas de coordinación y organización con agentes internos y externos.

3. Reuniones con equipo interno El tiempo utilizado en reuniones internas, es visto por algunos directivos como excesivo, ya que no les permite, como se mencionó, invertir tiempo en la generación de redes con otras entidades.

4. Reuniones con externos a la organización El tiempo usado para relacionarse con externos a la organización, es un tiempo "insuficiente", para algunos de los directivos, ya que les gustaría que fuera más extenso de lo que es efectivamente.

\section{Uso del tiempo en funciones sustantivas}

1. Gestión de prensa y vocería El uso del tiempo en temas de prensa y vocería, es diferenciado según en Servicio o Ministerio al que pertenezcan. Mientras en algunos casos esta función ocupa una parte de su tiempo, en otros casos, el porcentaje de tiempo es mínimo o nulo.

2. Gestionar el presupuesto (priorizar y asignar recursos) La mayoría de los directivos no hace mención al uso del tiempo en gestión de presupuestos, por lo que se puede entender que ésta función les ocupa un tiempo no muy importante. Sin embargo, uno de los directivos, menciona que ocupa el $60 \%$ de su tiempo en esas tareas, debido a que es parte de su "negocio".

3. Diseñar la organización (estructuras, procesos, planes) En el caso de algunos directivos, hay un tiempo alto dedicado al diseño o "rediseño" de la organización, lo cual depende del momento institucional por el cual pase el Servicio, y de las mejoras que se quiera hacer en éste.

4. "Apagar incendios" internos y negociar con asociaciones de funcionarios En general, el tiempo usado para negociar con asociaciones de funcionarios, está bien delimitado, y va desde dos reuniones mensuales a una reunión cada dos meses. Las estrategias desarrolladas por estos directivos, en cuanto a procurar que la tarea de relacionarse con gremios y asociaciones esté sistematizada o delegada en un principio en un encargado de relaciones laborales.

Asimismo se destaca que existe un número importante y mayoritario de "incendios" que son evitables mediante el diseño de buenos sistemas y estrategias de contención, para que no escalen hasta la Dirección.

5. Posicionar a la institución, gestión política y gestión de redes Aunque en muchos casos, se menciona la importancia de ejercer coordinaciones con otras entidades, personas y en general, crear redes, una vez más, ese tiempo se ve disminuido por tener que ejercer otras tareas al interior del mismo organismo, lo que les dificulta su trabajo "hacia afuera".

6. Pensar estratégicamente Si bien, no se cuantificó en términos de porcentaje, la mayoría de los directivos plantearon que en un cargo como este, es necesario tener un tiempo para pensar estratégicamente, tiempo que se ve disminuido por otras funciones de orden operativo.

\section{Conclusiones}

Las primeras aproximaciones dieron cuenta de actividades operativas que ocupaban parte importante del tiempo de los directivos públicos, mencionando explícitamente la firma de documentos que para algunos ocupa alrededor de 2 horas al día. La encuesta denota una burocratización de la función pública: $53 \%$ de los ADP creen que debiesen disminuir el tiempo que le dedican a firmar y/o visar documentos. Esta actividad constante y demandante, es vital para el funcionamiento del Servicio dado los marcos normativos vigentes, sin embargo desde la óptica de la eficiencia es muy cara esta actividad para la gestión del Estado.

En la misma línea los correos electrónicos laborales, son un fenómeno creciente dentro de las actividades diarias ineludibles para cualquier directivo.

La gestión de agenda es fundamental, se requiere un gran apoyo de los sistemas de información en línea para ello, así como los soportes de recursos físicos y humanos que permiten hacer más eficientes las actividades. La práctica directiva se juega en la cuantificación y dedicación de los tiempos según las actividades que debe abordar, distinguiendo entre actividades puntuales, cortas, reuniones acotadas o extensas, y aquellas de representación.

Respecto de las competencias interpersonales para liderar personas y grupos de trabajo es fundamental contar con buenos equipos y la capacidad de delegar en ellos y solicitar tareas como función 
sustantiva. Como producto de estos factores, se dispondrá de más tiempo por parte del directivo. Fundamentalmente en el equipo cercano debe generarse como práctica directiva un clima de confianza, que se va robusteciendo mediante las reuniones periódicas, función que fue altamente destacada como aquella que más tiempo le dedica el directivo en su jornada regular con un $19 \%$ y más aún el $54 \%$ quiere aumentar ese tiempo dedicado.

No obstante lo anterior, una buena práctica para ahorrar tiempo, es tener una metodología para hacer las reuniones. La reunión es un proceso, en que hay un cliente (en general el que cita), un producto a obtener, tiempo, recursos, etc. Todos los elementos de un proceso se encuentran en una reunión. El directivo que conduce una reunión, lleva este proceso, que debe llegar a resultados en un tiempo determinado. Las mejores reuniones, las hacen directivos con habilidades en la gestión de los procesos y con habilidades blandas. Tal como no se puede ejecutar o gestionar un proceso mal o no diseñado, una reunión no preparada, será lo más probable deficiente. Las reuniones hay que diseñarlas.

En cuanto a las competencias interpersonales para liderar personas, se devela que "seleccionar personas" es aquella donde los Altos Directivos Públicos se sientes más débiles. Esta información se condice con la etapa exploratoria y lo comentado por los directivos en el focus group realizado. También al menos un cuarto de los encuestado se declara medianamente conforme con su performance en "enseñar/servir de mentor/entrenar", competencia clave a la hora de ser un "buen jefe" para los funcionarios públicos.

Los imprevistos ocurren con frecuencia y hay que estar preparados. Estos pueden estar en el marco de las situaciones cotidianas de las funciones directivas o bien ser derechamente "incendios" cuya frecuencia y modo de resolución es incierto en un porcentaje importante del tiempo. En la misma línea, las dinámicas de los días son muy distintas y eso incide en la práctica directiva. Los días "no tranquilo", existe la sensación de "no estar trabajando", ante las contingencias e imprevistos que van apareciendo; mientras los días tranquilos le permiten al directivo hacer un uso más razonado de su tiempo en función de sus prioridades estratégicas institucionales.

Hay organizaciones que viven en permanente "incendio", en las cuales el directivo debe impulsar las siguientes prácticas, dentro de lo posible: (i) aprender de lo que hacen; (ii) despejar las incertidumbres de las responsabilidades asociadas; y (iii) evitar las duplicaciones. Los bomberos "no piensan" sino que actúan, pues el incendio no da tiempo para pensar.
Una organización en permanente incendio, es una organización que no piensa, no reflexiona, es decir, no se pregunta por los fundamentos de lo que hace.

Una práctica directiva interesante que se levantó, es el hecho de tomarse el tiempo por parte del directivo para preparar las reuniones, leer documentos, entender de lo que se va a hablar en instancias venideras de la agenda. Esto es una capacidad que se va desarrollando en el tiempo y pone al directivo en un pie de mayor seguridad y entendimiento, dentro de sus competencias interpersonales.

El manejo de la gestión de personas es un tema delicado dentro de las organizaciones y el directivo debe tener tacto para abordarlas. Al momento de relacionarse con funcionarios y gremios, existen ciertas competencias o estrategias que deben desplegar los directivos para poder tener un mejor diálogo. Por un lado, un ambiente grato y de cercanía se esboza como algo muy útil. Asimismo, es importante que los funcionarios entiendan las condiciones objetivas del Servicio y que adquieran la confianza en el directivo tomando en cuenta sus peticiones. Esta combinación es vislumbrada como algo de suma importancia al momento de mantener buenas relaciones. Tras ello se evidencia una clara práctica directiva, en la forma de hacer las cosas.

Existe una clara priorización de las funciones sustantivas más relevantes para los directivos de primer nivel jerárquico, a las cuales no hay que disminuir el esfuerzo físico y mental en ningún sentido:

\section{- Pensar estratégicamente.}

- Analizar información para la gestión.

- Posicionar a la institución, negociar con externos, gestión política y gestión de redes.

El pensamiento estratégico es un punto fundamental en la tarea que los atañe como Altos Directivos Públicos, y se destaca que tener incorporada esta práctica directiva es un requerimiento fundamental para ser un interlocutor efectivo entre el interior y exterior de la organización.

También aparece como necesidad tener una buena vocería y gestión de prensa. En general la relación con la ciudadanía y los usuarios es baja en tiempo y a un $47 \%$ le gustaría aumentar esa cifra. Probablemente las contingencias impiden la dedicación a la gran parte de los Altos Directivos Públicos les gustaría tener.

Queda claro que aún hay un importante desafío en gestión política de los directivos para con sus stakeholders. Este es uno de los aspectos que hace distinto a los directivos públicos en cuanto a 
su mandante en relación al sector privado, entre otras cosas. En las competencias relativas al sector público es donde se hace notar una mayor diferencia entre lo declarado por parte de los distintos niveles jerárquicos. En todas las competencias evaluadas, el nivel I aparece con capacidades y habilidades más desarrolladas para enfrentarse a los desafíos del sector público: la gestión política, el manejo de los medios, los gremios, la rendición de cuentas, entre otros.

Un punto muy interesante que cruzó el tema de la gestión efectiva, tuvo que ver con la discusión que se sostuvo relativo rol técnico versus un rol político que tiene que tener un Alto Directivo Público. Si bien se resguarda la importancia del rol técnico, es imposible mantenerse en el sistema público sin tener manejo político.

\section{Recomendaciones}

El intercambio de experiencias como herramienta para aprender modos de desempeñar la función directiva, es una práctica entre directivos que salió comentada tanto en la encuesta como en el focus group. En otras palabras el mentoring juega un rol clave para la agregación de valor de las prácticas directivas entre directivos públicos. Desde la literatura, la transferencia de buenas prácticas es un tópico vanguardista que ha entrado con fuerza en los Estados más avanzados, en el marco de modelos de innovación pública colaborativa, como lo probaron Willem y Buelens (2007), con el propósito de estudiar los factores relevantes en el proceso de compartir conocimiento durante episodios de cooperación en el sector público de Bélgica. Para el caso de los directivos públicos chilenos, que declaran vivir la "soledad" en el cargo, este método de transferencia de buenas prácticas directivas, podría ser una política que impulse el Servicio Civil, a través de su Subdirección de Desarrollo de las Personas. Una fuente de exploración e insumos para el diseño de la política, es saber si en el sector privado existen experiencias similares de traspaso de prácticas a nivel gerencial.

Los primeros avances de estas instancias deberían apuntar a desarrollar mayores competencias en aquellas áreas donde los mismos directivos se sienten con menos manejo y que en muchas ocasiones se repite el fenómeno, tal como lo mostró la encuesta. Ejemplos de ello son los "incendios que hay que apagar", donde se podría evitar que se inicien nuevos focos anteponiéndose con experiencia de otros Servicios antes de que se extienda el fuego; también hay problemáticas comunes en la relación con las asociaciones de funcionarios y prácticas compartibles que no se están diseminando.

Otro aspecto recurrente tiene que ver con el rol del Servicio Civil en la etapa de inducción. Se debe generar una instancia apropiada entendiendo los tiempos del directivo y apuntando en forma medianamente personalizada, para lograr efectividad en un plazo relativamente corto de tiempo. Algunas voces apelan por hacer de este procedimiento algo obligatorio, al menos para aquellos que efectivamente están llegando al mundo público por primera vez ya que los códigos implícitos son diferentes.

A su vez, se debe avanzar en programas de acompañamiento y entrenamiento para directivos superiores de la administración del Estado, incorporando estudios y conocimiento para apoyar la gestión de los directivos.

En términos del marco normativo, el país debería avanzar en reformas que hagan más eficientes las labores de los Altos Directivos Públicos. Por ejemplo, en una modernización universal de firmas electrónicas que permitiría bajar los desorbitantes tiempos que los directivos pasan firmando o visando documentos.

La distribución del tiempo, también tiene que ver con el tipo de profesionales con el que se cuenta, lo que obliga muchas veces a suplir falencias y competencias que debieran estar en los profesionales. Dada las dificultades de la administración pública para capacitar y reducir brechas, desvincular o mover a profesionales de puestos, resulta un escenario complejo que afecta el desempeño de funciones y la cantidad de tiempo que se le dedica a la organización para sacar adelante las metas, por parte del directivo público. Es misión del Servicio Civil, hacerse cargo de una política integral de la gestión de personas que se involucre los factores ya nombrados.

\section{Referencias}

Barzelay, M. (1998). Atravesando la burocracia: una nueva perspectiva de la administración pública. Fondo de Cultura Económica, México D.F.

Centro de Políticas Públicas UC (2013). Informe Final. Convenios de Desempeño. Rediseño de los convenios de desempeño de los altos directivos públicos. Report.

Drucker, P. (2001). Lo esencial de Drucker: Lo mejor de lo escrito sobre gerencia durante 60 años. Granica S.A., Buenos Aires.

Escobar, J. y Bonilla, F. (2009). Grupos Focales: 
Una guía conceptual y metodológica. Cuadernos Hispanoamericanos de Psicología, IX(1):51-67.

Etkin, J. (2005). Gestión de la Complejidad en las Organizaciones. Granica S.A., Buenos Aires.

Evans, P. y Rauch, J. (1999). Bureaucracy and growth: a cross national analysis of the effects of Weberian state structures on economic growth. American Sociological Review, 64(5):748-765.

Kotter, J. (1995). Leading Change: Why Transformation Efforts Fail. Harvard Business Review.

Lambertini, G. y Silva, E. (2009). Hacia un perfeccionamiento del Sistema de Alta Dirección Pública. En Un mejor Estado para Chile. Propuestas de modernización y reforma. Andros Impresores, Santiago.

Mintzberg, H. (1975). The Manager's Job: Folk Role and Fact. Harvard Business Review.

Mintzberg, H. (2009a). 29 Days of managing. Pearson Education.

Mintzberg, H. (2009b). Managing. Norma, San Francisco.

Morales, P. (2012). Tamaño necesario de la muestra: Â¿Cuántos sujetos necesitamos?
Shepherd, G. (1999). Administración Pública en América Latina y el Caribe: En busca de una paradgima de reforma. En Losada i Marrodi $i \frac{1}{2} \mathrm{n}$, C., editor, $\hat{A}_{\dot{ }}$ De burócratas a gerentes? Las ciencias de la gestión aplicadas a la administración del Estado, pp. 69-106. Banco Interamericano de Desarrollo, Washington, DC.

Valdés, S. (2001). Contratos y Remuneraciones de Altos Directivos Públicos. Centro de Estudios Públicos, Santiago.

Waissbluth, M., Inostroza, J., Acuña, E., y Avendaño, C. (2008). La Gestión de Organizaciones y Programas Públicos en Chile: Análisis de 28 casos para e implicancia para la Reforma del Estado. Technical report, Santiago.

Willem, A. y Buelens, M. (2007). Knowledge Sharing in Public Sector Organizations: The Effect of Organizational Characteristics on Interdepartmental Knowledge Sharing. Journal of Public Administration Research and Theory, 17(4):581606 .

World Bank (1997). Colombia: Paving the Way for a Results-Oriented Public Sector. A World Bank Study. Washington D.C. 